\title{
Calibration and Operation of a Bistatic Real-Aperture Polarimetric-Interferometric Ku- Band Radar
}

\section{Journal Article}

Author(s):

Stefko, Marcel (1D; Frey, Othmar (1); Werner, Charles; Hajnsek, Irena

Publication date:

2022

Permanent link:

https://doi.org/10.3929/ethz-b-000528527

\section{Rights / license:}

Creative Commons Attribution 4.0 International

Originally published in:

IEEE Transactions on Geoscience and Remote Sensing 60, https://doi.org/10.1109/tgrs.2021.3121466 


\title{
Calibration and Operation of a Bistatic Real-Aperture Polarimetric-Interferometric $\mathrm{Ku}-$ Band Radar
}

\author{
Marcel Stefko ${ }^{\circledR}$, Student Member, IEEE, Othmar Frey ${ }^{\circledR}$, Senior Member, IEEE, \\ Charles Werner ${ }^{\circledR}$, Life Senior Member, IEEE, and Irena Hajnsek ${ }^{\circledR}$, Fellow, IEEE
}

\begin{abstract}
This article presents the bistatic operation mode and the performance analysis of KAPRI, a terrestrial frequency-modulated continuous-wave (FMCW) Ku-band polarimetric radar interferometer capable of acquiring bistatic fullpolarimetric datasets with high spatial and temporal resolution. In the bistatic configuration, the system is composed of two independently operating KAPRI devices, one serving as a primary transmitter and receiver and the other as a secondary receiver. The secondary bistatic dataset is affected by possible offsets between the two devices' reference clocks, as well as distortions arising from the bistatic geometry. To correct for this, we present a two-chirp bistatic FMCW signal model, which accounts for the distortions, and a reference chirp transmission procedure, which allows correcting the clock offsets in the deramped signal time domain. The second challenge of operation of a bistatic polarimetric system is polarimetric calibration since it is not possible to employ purely monostatic targets such as corner reflectors. For this purpose, we developed a novel active calibration device Variable-Signature Polarimetric Active Radar Calibrator (VSPARC), designed for monostatic and bistatic calibration of all polarimetric channels. VSPARC and its associated novel polarimetric calibration method were then used to achieve full calibration of both KAPRI devices with polarimetric phase calibration accuracy of $20^{\circ}$ and 30-dB polarization purity in field conditions. This article thus presents a complete measurement configuration and data processing pipeline necessary for synchronization, coregistration, and polarimetric calibration of bistatic and monostatic datasets acquired by a real-aperture FMCW radar.
\end{abstract}

Index Terms-Active radar calibrator, bistatic radar, polarimetric calibration, real-aperture radar.

\section{INTRODUCTION}

B ISTATIC radar refers to a configuration of radar systems where the transmitter $(\mathrm{Tx})$ and receiver $(\mathrm{Rx})$ are

Manuscript received June 4, 2021; revised September 7, 2021; accepted October 4, 2021. Date of publication October 19, 2021; date of current version February 8, 2022. (Corresponding author: Marcel Stefko.)

Marcel Stefko is with the Chair of Earth Observation and Remote Sensing, Institute of Environmental Engineering, ETH Zürich, 8093 Zürich, Switzerland (e-mail: stefko@ifu.baug.ethz.ch).

Othmar Frey is with the Chair of Earth Observation and Remote Sensing, Institute of Environmental Engineering, ETH Zürich, 8093 Zürich, Switzerland, and also with GAMMA Remote Sensing AG, 3073 Gümligen, Switzerland.

Charles Werner is with GAMMA Remote Sensing AG, 3073 Gümligen, Switzerland.

Irena Hajnsek is with the Chair of Earth Observation and Remote Sensing, Institute of Environmental Engineering, ETH Zürich, 8093 Zürich, Switzerland, and also with the Microwaves and Radar Institute, German Aerospace Center, 82234 Weßling, Germany.

Digital Object Identifier 10.1109/TGRS.2021.3121466 spatially separated. It is of research interest because of its complementary properties to the monostatic case, such as access to a larger number of polarimetric parameters [1], [2], recovery of 3-D displacement vectors [3], [4], possibility of long-baseline single-pass interferometric measurements [5], increased radar cross section of certain targets such as the sea surface [6], retrieval of biophysical parameters [7]-[9], and also military surveillance applications [10]-[12].

Flexibility and lower costs of terrestrial devices make them especially useful for exploratory acquisitions, as well as development and validation of new observation methods and processing algorithms, upon which airborne and spaceborne missions can be designed and implemented. Furthermore, for small-scale applications, terrestrial sensors can offer denser temporal sampling (as opposed to spaceborne sensors) and longer observation periods (as opposed to airborne sensors) while keeping costs low.

\section{A. State of the Art}

1) Bistatic Radar for Earth Observation: The field of bistatic radar for monitoring of the natural environment has not been as thoroughly explored as its monostatic counterpart since the requirement of physical separation between the transmitter and the receiver introduces additional technical and operational challenges [13]. Continued interest of the scientific community in this area is reflected in past proposals of bistatic satellite mission concepts [14]-[17], as well as proposals currently in evaluation such as Tandem-L [3], Harmony [18], and ROSE-L [19]. The Shuttle Radar Topography Mission [20] employed a single-pass interferometry configuration, where a considerable bistatic baseline between receiver antennas on a single platform was achieved through deployment of an extensible mast. TanDEM-X [21] is the first and currently only mission to demonstrate spaceborne bistatic synthetic aperture radar (SAR) measurements using two completely separate platforms and has a bistatic angle $\beta$ on the order of $1^{\circ}$ or less in nominal operation.

Several experimental bistatic campaigns using airborne SAR systems had been carried out in the past two decades, such as the ONERA-DLR bistatic SAR experiment [5], MetaSensing's BelSAR campaign [22], [23], and hybrid bistatic experiments between a spaceborne transmitter (TerraSAR-X) and an airborne receiver (F-SAR [24] and PAMIR [25]). 
Ground-based measurements offer more flexibility in terms of temporal coverage and system configuration, such as a systematic variation and sampling of different bistatic angles. Several experimental bistatic terrestrial radar devices employing aperture synthesis were built recently [4], [26]-[28], as well as multiple passive bistatic SAR (PB-SAR) receivers that use orbiting satellites as transmitters of opportunity [29]-[34].

There is a relative lack of available polarimetricinterferometric bistatic datasets with large bistatic angles since most of the available systems either operate in the small bistatic angle mode for single-pass InSAR purposes [21], [23], are of one-off experimental nature [5], [24], [25], can only operate in very constrained geometry [26], [27], [29]-[32], or do not have full-polarimetric capabilities [4], [29], [30], [32]. Such datasets are, however, vital for development, testing, and verification of future bistatic spaceborne mission concepts and model-based inversion procedures. Terrestrial radars that are capable of acquiring polarimetric-interferometric datasets at a variety of bistatic angles such as the bistatic KAPRI provide a possibility to perform many of these required measurements.

2) Bistatic Real-Aperture Radar: Partly due to its potential surveillance applications, bistatic real-aperture radar geometry and associated parameters, such as signal intensity, range, clutter cell area, and others, have been extensively analyzed in the literature [10], [35], [36].

3) Polarimetric Calibration: In the monostatic case, polarimetric calibration of radar systems is usually performed using trihedral corner reflectors, which serve as a robust and passive target with a high radar cross section for radiometric and polarimetric calibration [37]. For cross-polarized channels, usually, a supplemental target with a strong cross-polarizing signature [38], [39] or alternative approaches such as the reciprocity principle are utilized [37], [40].

Neither the trihedral corner reflector nor the reciprocity principle is usable in the bistatic case. Bistatic calibration is thus performed via other approaches, such as a modified dihedral with a varying opening angle [41], [42], a crosspolarizing cylinder [43], or an active calibrator [5], [44], [45].

\section{B. KAPRI: A Real-Aperture Polarimetric-Interferometric FMCW Radar With Bistatic Capabilities}

Monostatic operation mode and the polarimetric calibration of KAPRI (Ku-band Advanced Polarimetric Radar Interferometer) - a real-aperture, frequency-modulated continuous-wave (FMCW) radar-have been introduced in [40], with application to observation of natural environments in [46] and [47]. It was built by Gamma Remote Sensing and is an extension of the GPRI [48]-[51] with fully polarimetric capabilities and a custom hardware extension that allows chirp synchronization. By employing two KAPRI devices, one as a transmitter and the other as a receiver, and increasing the beamwidth of the receiver device's antennas, it is possible to perform bistatic acquisitions while preserving the high flexibility of the acquisition geometry. To the best of our knowledge, KAPRI is the first realaperture, full-polarimetric, interferometric, and bistatic radar system capable of monitoring areas kilometers in size with meter-scale resolution.

\section{Contributions of This Article}

The following procedures, which are necessary in order to fully allow bistatic operation and acquisition of calibrated bistatic single look complex (SLC) datasets, are described in this article:

1) synchronizing the acquisition start times and frequency sweeps of both radars using a synchronization link;

2) compensating for relative drift of local oscillators (LOs) and resulting frequency/bandwidth/phase offsets;

3) modeling the bistatic geometry in order to consider the elliptical shape of bistatic iso-range lines, and the antenna gain of the bistatic transmit and receive antennas;

4) calibrating individual polarimetric channels of both devices-this required development of a novel calibration target capable of calibration of both co- and crosspolarized channels in both monostatic and bistatic acquisition modes, as well as application of an appropriate calibration method.

These procedures are then validated via analysis of the following:

1) the synchronization signal behavior in terms of its phase and frequency;

2) polarization purity and phase retrieval accuracy of both devices;

3) the polarimetric signature of the novel active calibration device;

4) polarimetric calibration coefficients retrieved for both devices using the novel active calibration method and their comparison to coefficients retrieved using the previously validated calibration procedure using passive corner reflectors described in [40].

\section{Outline}

Section II-A presents the geometrical configuration employed for bistatic KAPRI acquisitions and the additional instrumentation required for bistatic operation. In Sections II-B and II-C, a signal model that considers the two-chirp nature of the bistatic measurements is developed, and Section II-D shows how these time-related oscillator distortions are compensated by a synchronization link. Section II-E analyzes the effect of the synchronization link and lower antenna gain on phase retrieval accuracy of the bistatic receiver. Section II-F describes a procedure that aligns the bistatic dataset acquired in an elliptical geometry with the polar geometry of the monostatic dataset and compensates for varying resolution cell size. Possible off-center gain corrections in case of uneven horn antenna patterns are described in Section II-G. Section II-H describes a novel calibration target well-suited for calibration of bistatic polarimetric-interferometric systems, and Section II-I describes the polarimetric calibration method, which makes use of this novel target. Finally, the processing pipeline is summarized in Section II-J.

Several experiments were carried out to demonstrate certain features of bistatic KAPRI operation. Section III-A verifies the 
TABLE I

MAIN SYSTEM PARAMETERS OF KAPRI

\begin{tabular}{rcl}
\hline Parameter & Symbol & Value \\
\hline Start frequency & $f_{\mathrm{c}}$ & $17.1 \mathrm{GHz}$ \\
Chirp bandwidth & $B$ & up to $200 \mathrm{MHz}$ \\
Output power & $P_{\mathrm{t}}$ & $21.5 \mathrm{dBm}$ \\
Receiver noise figure & $F_{\mathrm{n}}$ & $3.1 \mathrm{~dB}$ at $290 \mathrm{~K}$ \\
FMCW chirp duration & $\tau$ & between $250 \mu \mathrm{s}$ and $16 \mathrm{~ms}$ \\
Range sample spacing & $\delta^{\text {rs }}$ & $0.75 \mathrm{~m}$ \\
Primary (P) azimuth beamwidth & $\delta_{\mathrm{P}}^{\theta}$ & $0.5^{\circ}$ \\
Primary (P) elevation beamwidth & $\delta_{\mathrm{P}}^{\varepsilon}$ & $>31^{\circ}$ \\
Secondary (S) azimuth beamwidth & $\delta_{\mathrm{S}}^{\theta}$ & $12^{\circ}$ \\
Secondary (S) elevation beamwidth & $\delta_{\mathrm{S}}^{\varepsilon}$ & $24^{\circ}$ \\
Clock frequency & $f_{\text {clock }}$ & $100 \mathrm{MHz}$ GPS-disciplined \\
\hline
\end{tabular}

Note: Beamwidth values correspond to one-way HPBW.

signal model by analyzing the predicted connection between the slow time behavior of the reference signal's range position and phase. Section III-B analyzes the polarimetric signature of the novel calibration target. Section III-C analyzes polarization purity with respect to the requirements of the chosen polarimetric calibration method, as well as effects of antenna gain on phase retrieval accuracy. Section III-D verifies the polarimetric calibration method by comparing the resulting calibration parameters with the parameters returned from the monostatic calibration method introduced in [40]. Finally, Section III-E provides a visualization of key steps of the bistatic processing pipeline.

Section IV discusses the geometric features of bistatic KAPRI datasets, properties of the reference signal, validity of the novel calibration method and its advantages, as well as possible applications of bistatic KAPRI within the framework of Earth observation. A conclusion is given in Section V.

\section{Methods}

\section{A. Bistatic KAPRI Configuration}

The bistatic configuration of KAPRI consists of two devices-the monostatic transmitter-receiver $\mathrm{P}$ (primary) and a bistatic receiver $\mathrm{S}$ (secondary). The antennas of the primary monostatic KAPRI device are mounted on a single rotating tower with two alternating transmitting and four receiving slotted-waveguide antennas with a half-power beamwidth (HPBW) of approximately $0.5^{\circ}$ in azimuth. To obtain a bistatic configuration, the secondary tower employing a set of four receiving antennas with a gain pattern HPBW of $12^{\circ}$ in azimuth is set up. The addition of the passive bistatic receiver has the benefit that two datasets-one monostatic and one bistatic - are acquired during each acquisition. This creates possibilities to naturally combine and extend the wellknown monostatic polarimetric imaging mode into the bistatic mode.

Key system parameters of the bistatic KAPRI configuration are summarized in Table I. The wider antenna beamwidth of the bistatic receiver was chosen for practical reasons-at any given time, only the targets that are located in the intersection of the Tx and Rx antenna beams can be imaged (see Fig. 1). In the bistatic geometry, narrow-beam receiver antennas would

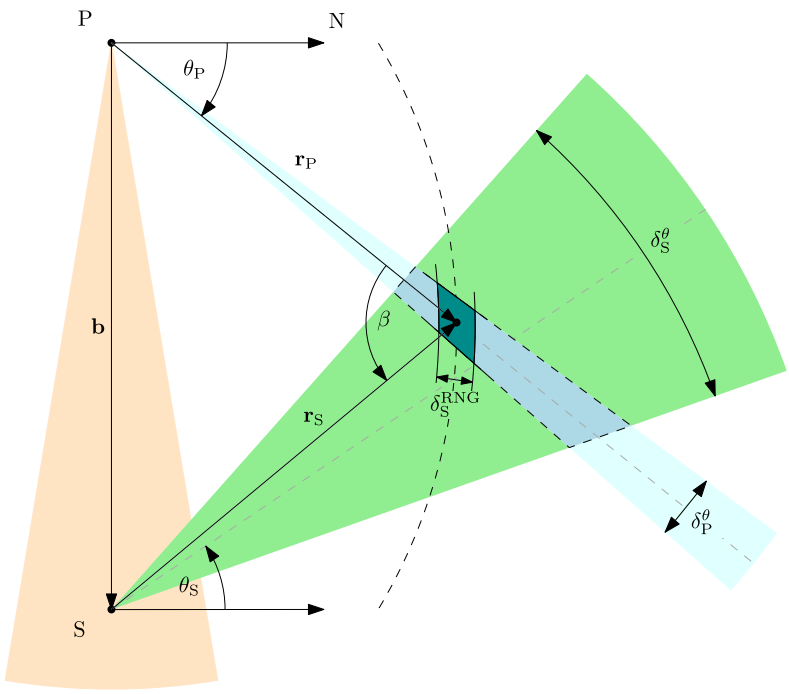

Fig. 1. Bistatic KAPRI configuration. In the bistatic north-referenced coordinate system, the secondary device (S) is at the $90^{\circ}$ azimuth coordinate with respect to the primary device $(\mathrm{P})$. The dashed curve marks the elliptical isorange line. Azimuth resolution is provided by the narrow aperture of the rotating primary antennas (blue), while the pattern of static wide-aperture secondary receiver horn antennas (green) defines the total covered area. The teal-colored area defines one resolution cell. The light-brown colored synchronization beam transmits the bistatic reference signal directly from the primary to the secondary device.

TABLE II

EXAMPLES OF RANGE $\left(\delta^{\mathrm{RNG}}\right)$ AND AZIMUTH $\left(\delta^{\mathrm{AZM}}\right)$ RESOLUTIONS OF Monostatic Primary (P) AND Bistatic SECONDARY (S) KAPRI With VARYing Values of Range From Primary DeVice $\left(r_{\mathrm{P}}\right)$ and Bistatic Angle $(\beta)$. Resolution Values CORRESPOND TO - 3 -dB PEAK WidTH

\begin{tabular}{cccccc}
\hline$r_{\mathrm{P}}$ & $\beta$ & $\delta_{\mathrm{P}}^{\mathrm{RNG}}$ & $\delta_{\mathrm{S}}^{\mathrm{RNG}}$ & $\delta_{\mathrm{P}}^{\text {AZM }}$ & $\delta_{\mathrm{S}}^{\text {AZM }}$ \\
\hline $400 \mathrm{~m}$ & $0^{\circ}$ & $0.95 \mathrm{~m}$ & $0.95 \mathrm{~m}$ & $2.69 \mathrm{~m}$ & $3.49 \mathrm{~m}$ \\
$400 \mathrm{~m}$ & $30^{\circ}$ & $0.95 \mathrm{~m}$ & $1.02 \mathrm{~m}$ & $2.69 \mathrm{~m}$ & $3.49 \mathrm{~m}$ \\
$400 \mathrm{~m}$ & $120^{\circ}$ & $0.95 \mathrm{~m}$ & $3.80 \mathrm{~m}$ & $2.69 \mathrm{~m}$ & $3.49 \mathrm{~m}$ \\
$6000 \mathrm{~m}$ & $0^{\circ}$ & $0.95 \mathrm{~m}$ & $0.95 \mathrm{~m}$ & $40.3 \mathrm{~m}$ & $52.4 \mathrm{~m}$ \\
$6000 \mathrm{~m}$ & $30^{\circ}$ & $0.95 \mathrm{~m}$ & $1.02 \mathrm{~m}$ & $40.3 \mathrm{~m}$ & $52.4 \mathrm{~m}$ \\
$6000 \mathrm{~m}$ & $120^{\circ}$ & $0.95 \mathrm{~m}$ & $3.80 \mathrm{~m}$ & $40.3 \mathrm{~m}$ & $52.4 \mathrm{~m}$ \\
\hline
\end{tabular}

provide higher gain, but the beam intersection - and thus the imaged area size-would be prohibitively small. A beamwidth of $12^{\circ}$ for the bistatic receiver is a compromise between coverage and signal strength. Table II shows the range and azimuth resolutions of both devices in multiple example geometric configurations.

In general, bistatic experiments require a synchronization procedure, preferably using a dedicated built-in link (such as in case of TanDEM-X [52]). KAPRI also employs such a link-Fig. 2 shows the reference chirp transmission method: a directional coupler is used to transmit approximately $10 \%$ of chirp power directly from the primary to the secondary device. This directly transmitted signal is used to correct phase and frequency offsets, as described in Section II-C.

\section{B. Bistatic KAPRI FMCW Signal Model}

KAPRI is using a deramp-on-receive FMCW radar architecture [53]. The monostatic signal model is described in [40], 


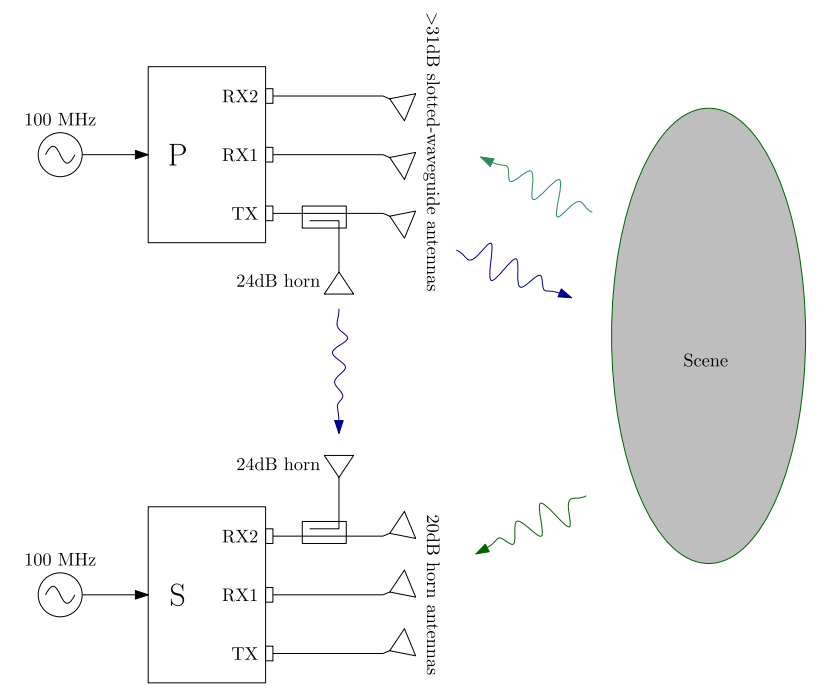

Fig. 2. Simplified antenna configuration. Each device is driven by its own local oscillator (LO), and these two LOs drift with respect to one another. In order to correct this phase drift, directional couplers are used to transmit a part of the signal directly from the primary to the secondary. This signal then serves as a reference for phase drift correction. The nominal gain and type of each antenna are marked in the figure.
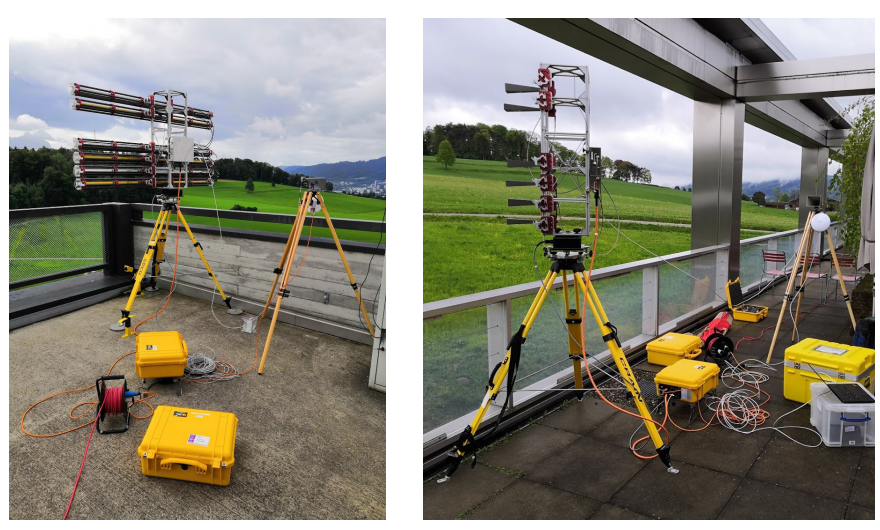

Fig. 3. Bistatic KAPRI device configuration (left: primary device $(P)$ equipped with narrow-beam slotted-waveguide antennas and right: secondary receiver device (S) equipped with horn antennas). Reference link horn antennas are placed on tripods on the right side of the two images, together with long-range Wi-Fi antennas for remote device control.

and this section describes the bistatic signal model and acquisition mode. The measurement geometry is shown in Fig. 1, and the device configuration depicting the antennas used and the reference chirp transmission is shown in Fig. 2. Photographs of the deployed devices are shown in Fig. 3.

The two devices are not perfectly synchronized-varying conditions at operation locations, as well as unavoidable manufacturing differences in parameters such as exact oscillator base frequency, cause differences between their frequencymodulated chirps. This can be addressed by modeling each device's linear FM chirp with its own start frequency, bandwidth, and sweep start time.

The primary device transmits a linearly modulated signal $s_{\mathrm{pt}}(t)$ of duration $\tau$ with bandwidth $B$ and start frequency $f_{c}$

$$
s_{\mathrm{pt}}(t)=e^{j 2 \pi\left(f_{c} t+\frac{\gamma}{2} t^{2}\right)}
$$

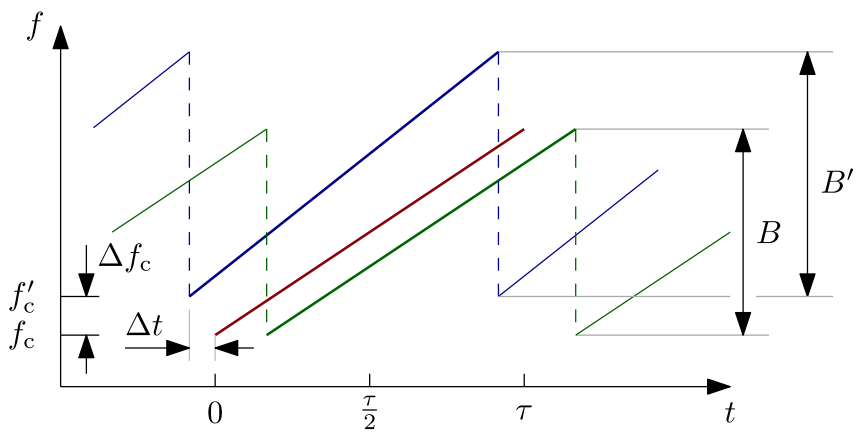

Fig. 4. Time-frequency diagram of the transmitted $s_{\mathrm{pt}}$ (dark red), received $s_{\text {sr }}$ (green), and receiver reference $s_{\text {st }}$ (blue) chirps. Start frequency offset $\Delta f_{c}$ and bandwidth offset $\Delta B$ between transmitter and receiver chirps cause range offset and peak smearing within the range-compressed data, respectively. While the start times of chirps are synchronized via GPS PPS signals at acquisition start, due to clock speed offsets, a chirp start time offset $\Delta t$ accumulates over the course of the acquisition, causing additional range and phase drift of the deramped signal.

where $\gamma=B / \tau$ is the chirp rate. The signal is then scattered by a scatterer with complex reflectivity $\sigma$ and delayed by $p / c$, where

$$
p=r_{\mathrm{P}}+r_{\mathrm{S}}
$$

is the bistatic path length. The signal received at the secondary receiver $s_{\mathrm{sr}}(t)$ is then

$$
s_{\mathrm{sr}}(t)=\sigma e^{j 2 \pi\left(f_{c}\left[t-\frac{p}{c}\right]+\frac{\gamma}{2}\left[t-\frac{p}{c}\right]^{2}\right)} .
$$

The secondary device (i.e., bistatic receiver) generates its own linearly modulated chirp $s_{\mathrm{st}}(t)$ with bandwidth $B^{\prime}$ and start frequency $f_{c}^{\prime}$

$$
s_{\mathrm{st}}(t)=e^{j 2 \pi\left(f_{c}^{\prime}[t-\Delta t]+\frac{y^{\prime}}{2}[t-\Delta t]^{2}\right)}
$$

where $\Delta t$ describes the starting time offset between the two devices' chirps and $\gamma^{\prime}=B^{\prime} / \tau$. The time-frequency diagram of this signal model is shown in Fig. 4.

In the secondary device's receiver, the received bistatic signal $s_{\mathrm{sr}}$ is mixed with its own reference chirp $s_{\mathrm{st}}$, which removes the linear modulation and results in a deramped signal $s_{\mathrm{d}}$

$$
\begin{aligned}
s_{\mathrm{d}}(t) & =s_{\mathrm{sr}}(t)^{*} s_{\mathrm{st}}(t) \\
& =\sigma^{*} e^{j 2 \pi\left(\left[\frac{p y}{c}+\Delta f_{c}-\gamma^{\prime} \Delta t\right] t+\frac{p}{\lambda}-\frac{p^{2} \gamma}{2 c^{2}}+\frac{\Delta \gamma}{2} t^{2}-f_{\mathrm{c}}^{\prime} \Delta t+\frac{y^{\prime}}{2} \Delta t^{2}\right)}
\end{aligned}
$$

where $\lambda=c / f_{c}$ is the wavelength, $\Delta f_{c}=f_{c}^{\prime}-f_{c}$ is the start frequency offset between the two radars, $\Delta \gamma=\gamma^{\prime}-\gamma$ is chirp rate offset between the two radars, and the ${ }^{*}$ symbol represents the complex conjugate.

The six phase terms in the complex exponent in (5b) are as follows:

1) $2 \pi\left[p \gamma / c+\Delta f_{c}-\gamma^{\prime} \Delta t\right] t$-beat signal with beat frequency proportional to the signal travel path $p$, shifted by $\Delta f_{c}$ and $-\gamma^{\prime} \Delta t$ due to offset between the two radars' start frequencies and chirp start times, respectively;

2) $2 \pi p / \lambda$-two-way propagation phase;

3) $-\pi p^{2} \gamma / c^{2}$ — "residual video phase" (RVP) present also in the monostatic deramped signal [40]; 
4) $\pi \Delta \gamma t^{2}$-residual frequency ramp caused by chirp rate offset $\Delta \gamma$;

5) $-2 \pi f_{c}^{\prime} \Delta t$-dominant phase drift term caused by start time offset $\Delta t$

6) $\pi \gamma^{\prime} \Delta t^{2}$-second-order time offset term.

These terms are analyzed in more detail in Section II-C.

\section{Deramped Signal Analysis}

By noting the time dependence of the first term in the exponential of $(5 b)$, one can estimate the profile of the complex reflectivity of the observed scene $\hat{\sigma}(p, \theta)$ by taking the Fourier transform of $s_{\mathrm{d}}(t)^{*}$. Several corrections are, however, needed in order to achieve data coregistration, and polarimetric and interferometric calibration since $s_{\mathrm{d}}(t)$ contains additional terms as a result of possible mismatching chirp rate, start frequency, and LO frequency between transmitter and receiver devices.

1) Beat Signal: The beat signal term of (5b) has three components: the signal travel path component $p \gamma / c$ (the term of interest for ranging using FMCW radar), the frequency offset $\Delta f_{c}$, and start time offset $-\gamma^{\prime} \Delta t$. We define the total observed frequency offset $\Delta f$

$$
\Delta f=\Delta f_{c}-\gamma^{\prime} \Delta t
$$

This offset causes incorrect range determination for the observed scene. Furthermore, the value of this offset possibly changes on both short time scales of individual pulses (due to accumulation of start time offset $\Delta t$ ), as well as longer time scales (due to changes in $\Delta f_{c}$ and $\gamma^{\prime}$ due to variations in environmental conditions).

2) Two-Way Propagation Phase: The phase term

$$
\phi_{\text {prop }}=2 \pi \frac{p}{\lambda}
$$

is the quantity of interest for interferometric measurements since wavelength-scale changes in signal travel path $p$ cause considerable changes in the value of this term.

3) Residual Video Phase: The term

$$
\phi_{\mathrm{rvp}}=-\pi \frac{p^{2} \gamma}{c^{2}}
$$

is assumed to be constant for each target over the antenna aperture for the KAPRI configuration [40]. The value of this term varies with the signal travel path length, similar to the propagation phase term. We can define changes in signal phase $\Delta \phi$ due to a small change in signal path $\Delta p$ at total path $p$ as

$$
\begin{aligned}
\Delta \phi_{\text {prop }}(p, \Delta p) & =\frac{2 \pi(p+\Delta p)}{\lambda}-\frac{2 \pi p}{\lambda}=\frac{2 \pi \Delta p}{\lambda} \\
\Delta \phi_{\text {rvp }}(p, \Delta p) & =\cdots=-\frac{\pi \gamma}{c^{2}}\left(2 p \Delta p+\Delta p^{2}\right) .
\end{aligned}
$$

The ratio of magnitudes of these changes is then

$$
\left|\frac{\Delta \phi_{\mathrm{rvp}}}{\Delta \phi_{\text {prop }}}(p, \Delta p)\right|=\frac{\lambda \gamma}{2 c^{2}} \frac{2 p \Delta p+\Delta p^{2}}{\Delta p} \approx \frac{\lambda \gamma p}{c^{2}}=\frac{\gamma p}{f_{c} c} \text {. }
$$

For KAPRI parameters, the worst case estimate of this ratio is $\sim 10^{-4}$, and thus, the propagation phase term varies with $p$ orders of magnitude faster than the RVP term. Because of this, for most interferometric and polarimetric purposes, the effects of RVP can be neglected. If necessary, the RVP contribution can be trivially corrected by phase-shifting each range cell by the corresponding value of (8).

4) Residual Frequency Ramp: Reflected in term $\pi \Delta \gamma t^{2}$, bandwidth mismatch results in a residual low-frequency chirp component in the deramped signal, with peak frequency $\Delta \gamma \tau$. This residual chirp defocuses the compressed signal over a range of neighboring frequencies and thus degrades the spatial resolution.

5) Phase Drift Term: The fifth phase term in (5b)

$$
\phi_{\mathrm{d}}=-2 \pi f_{c}^{\prime} \Delta t
$$

is caused by the changing value of start time offset $\Delta t$. This offset accumulates in-between synchronizations via GPS PPS pulses, which resets $\Delta t$ back to 0 . We can compute the current value of $\Delta t$ at time $T$ since the last synchronization as

$$
\Delta t(T)=\int_{0}^{T} \frac{\Delta \tau\left(T^{\prime}\right)}{\tau} d T^{\prime}
$$

where $\Delta \tau\left(T^{\prime}\right)$ is the difference between the two devices' chirp lengths $\tau$ and $\tau^{\prime}$ at slow time $T^{\prime}$. The phase drift rate can then be calculated by taking the slow-time derivative of (11)

$$
\frac{d \phi_{\mathrm{d}}(T)}{d T}=-2 \pi f_{c}^{\prime} \frac{d \Delta t(T)}{d T}=-2 \pi f_{c}^{\prime} \frac{\Delta \tau(T)}{\tau} .
$$

The phase drift rate at any given moment is thus linearly proportional to the value of $\Delta \tau$. This phase drift can compromise interferometric measurements, since within $s_{\mathrm{d}}(t)$, the phase drift $\phi_{\mathrm{d}}$ is indistinguishable from changes in the geometric phase term $\phi_{\text {prop }}$ and needs to be corrected via the synchronization link.

6) Second-Order Phase Drift Term: Similar to the RVP term, the effects of second-order phase drift term

$$
\phi_{\mathrm{d}, 2}=\pi \gamma^{\prime} \Delta t^{2}
$$

are negligible compared to the previous term

$$
\frac{\phi_{\mathrm{d}, 2}}{\phi_{\mathrm{d}}}=-\frac{\gamma^{\prime} \Delta t}{2 f_{c}^{\prime}} .
$$

Experiments show that the value of $\Delta t$ in nominal operation is at most $100 \mathrm{~ns}$ at any time, corresponding to a worst case ratio of $\sim 10^{-5}$.

\section{Correction via Reference Signal Transmission}

Offset effects described in the previous section can be corrected in the data by transmitting a reference signal directly from primary transmitter to secondary receiver (see Fig. 2). This reference signal does not scatter off the scene and, at the receiver, has the form

$$
S_{\text {Sr-ref }}(t)=e^{j 2 \pi\left(f_{c}\left[t-\frac{b}{c}\right]+\frac{y}{2}\left[t-\frac{b}{c}\right]^{2}\right)}
$$

where $b$ is the length of the bistatic baseline. The corresponding deramped reference signal is

$$
\begin{aligned}
s_{\mathrm{d}-\text { ref }}(t) & =s_{\text {sr-ref }}(t)^{*} s_{\mathrm{st}}(t) \\
& =e^{j 2 \pi\left(\left[\frac{b_{y}}{c}+\Delta f\right] t+\frac{b}{\lambda}-\frac{b^{2} y}{2 c^{2}}+\frac{\Delta y}{2} t^{2}-f_{c}^{\prime} \Delta t+\frac{\gamma^{\prime}}{2} \Delta t^{2}\right) .}
\end{aligned}
$$


By multiplying the deramped signal $s_{\mathrm{d}}$ by the complex conjugate of the deramped reference signal $s_{\mathrm{d} \text {-ref }}$, one can compensate for the drift effects

$$
s_{\mathrm{d}}(t) s_{\mathrm{d}-\mathrm{ref}}(t)^{*}=\sigma^{*} e^{j 2 \pi\left(\frac{(p-b) y)}{c} t+\frac{p-b}{\lambda}-\frac{\left(p^{2}-b^{2}\right) y}{2 c^{2}}\right)}
$$

and by multiplying the expression in (18) by an additional oscillating term with frequency $\frac{b \gamma}{c}$, the corrected deramped signal $s_{\mathrm{d} \text {-corr }}$ is recovered

$$
\begin{aligned}
s_{\mathrm{d}-\mathrm{corr}}(t) & =s_{d}(t) s_{\mathrm{d}-\mathrm{ref}}(t)^{*} e^{j 2 \pi \frac{b y}{c} t} \\
& =\sigma^{*} e^{j 2 \pi\left(\frac{p y}{c} t+\frac{p-b}{\lambda}-\frac{\left(p^{2}-b^{2}\right) y}{2 c^{2}}\right)} .
\end{aligned}
$$

Analysis of this signal then enables interferometric, polarimetric, and bistatic radar cross section (RCS) measurements. In order to recover it, it is necessary to be able to isolate the reference signal $s_{\mathrm{d}-\mathrm{ref}}(t)$ from the total deramped signal $s_{\mathrm{d}}(t)$.

The reference signal can be either transmitted using its own channel or superimposed on the signal from the scene (e.g., by use of a directional coupler as in Fig. 2). Due to triangle inequality, any signals scattered from the scene will have a path delay longer than the directly transmitted reference chirp, i.e., $p>b$. This means that even when the reference chirp is not transmitted via a dedicated channel, the reference signal can be identified within the SLC as the peak with the shortest range distance. Provided that the scene area in the region where $p \approx b$ is relatively clutter-free, the deramped observed reference signal $\hat{s}_{\mathrm{d}-\mathrm{ref}}$ can be isolated by applying a window around the range-compressed signal $\mathcal{F}\left[\hat{s}_{\mathrm{d}}\right]$

$$
\hat{s}_{\text {d-ref }}(t)=\mathcal{F}^{-1}\left[\mathcal{F}\left[\hat{s}_{\mathrm{d}}\right] W\right]
$$

where $\mathcal{F}$ denotes the Fourier transform and $W$ is a window (e.g., a Hanning window) in the range-compressed signal domain centered around the range distance $b$ and wide enough to capture the reference signal even when it is offset in frequency by $\Delta f$ and spread out by bandwidth offset $\Delta \gamma$.

Having isolated $\hat{s}_{\mathrm{d} \text {-ref }}$, it is now possible to correct for the offset effects in the scene signal $\hat{s}_{\mathrm{d}}$ via multiplication in the deramped signal domain

$$
\hat{s}_{\mathrm{d}-\mathrm{corr}}(t)=\hat{s}_{\mathrm{d}}(t) \hat{s}_{\mathrm{d}-\mathrm{ref}}(t)^{*} e^{j 2 \pi \frac{b y}{c} t}
$$

where the final term with beat frequency $b \gamma / c$ ensures correct range positioning of the corrected signal. Finally, $\mathcal{F}\left[\hat{s}_{\mathrm{d}-\mathrm{corr}}(t)\right]$ yields the corrected range-compressed bistatic signal.

\section{E. Phase Retrieval Accuracy}

The bistatic acquisition configuration has an effect on phase retrieval accuracy of $\hat{s}_{\mathrm{d} \text {-corr }}$ in two ways.

1) The reduced gain of the secondary device's antennas (necessary to increase the beam pattern width) causes a reduction of signal-to-noise ratio (SNR) of scene signal $\hat{s}_{\mathrm{d}}$.

2) Any phase noise along the directly transmitted reference signal link affects $\hat{s}_{\mathrm{d} \text {-ref }}$.

The final phase variance can then be expressed as a sum of the variances of the two effects

$$
\sigma_{\phi}^{2}=\sigma_{\phi, \text { scene }}^{2}+\sigma_{\phi, \text { ref }}^{2}
$$

where $\sigma_{\phi, \text { scene }}$ is the phase noise contribution of the scene signal $\hat{s}_{\mathrm{d}}$ and $\sigma_{\phi \text {,ref }}$ is the phase noise contribution of the reference signal $\hat{s}_{\text {d-ref }}$.

We now investigate the contribution of the reference signal $\sigma_{\phi, \text { ref }}$. The variance of the phase noise of a signal is related to the SNR of the signal and in case of high SNR can be estimated as [54]-[56]

$$
\sigma_{\phi, \text { ref }}^{2}=\frac{1}{\mathrm{SNR}_{\mathrm{ref}}} .
$$

The exact requirement for value of $\sigma_{\phi \text {,ref }}$ depends on the particular application. For example, in order to achieve the value of phase noise standard deviation of the reference signal below $2^{\circ}$, we can determine the necessary SNR

$$
\sigma_{\phi, \text { ref }} \leq 2^{\circ} \Longrightarrow \mathrm{SNR}_{\mathrm{ref}} \geq 29.1 \mathrm{~dB} .
$$

The reference signal's SNR can be computed using the nominal parameters of the KAPRI system and applying the one-way radar equation [57], [58]

$$
\mathrm{SNR}_{\mathrm{ref}}=\frac{P_{t} \tau G_{t} G_{r} \lambda^{2}}{(4 \pi b)^{2} k T_{0} F_{n} L}
$$

where $P_{t}$ is the transmitter output power, $\tau$ is the transmitted pulselength, $G_{t}$ and $G_{r}$ are the gains of corresponding transmit and receive antennas, respectively, $L$ are the line losses, $b$ is the bistatic baseline, $k$ is Boltzmann's constant, $T_{0}$ is the system temperature, and $F_{n}$ is the system noise figure.

Taking the logarithmic form of (25), we can write

$$
\mathrm{SNR}_{\mathrm{ref}}^{\mathrm{dB}}=10\left[\log \frac{P_{t} \tau G_{t} G_{r}}{k T_{0} F_{n} L}+2 \log \frac{\lambda}{4 \pi b}\right] .
$$

The SNR value thus reduces/increases by $6 \mathrm{~dB}$ for each doubling/halving of the baseline length $b$ and increases/ reduces by $3 \mathrm{~dB}$ for each doubling/halving of the chirp length $\tau$.

To account for all system losses and noise sources along the signal path, we determined a reference SNR value for KAPRI experimentally by determining the intensity of the reference signal peak during an acquisition $I_{\text {ref }}$ and comparing it to the noise floor $I_{\text {noise }}{ }^{1}$

$$
\mathrm{SNR}_{\text {ref }}=\frac{I_{\text {ref }}}{I_{\text {noise }}} \approx 50 \mathrm{~dB} \text { at } b=950 \mathrm{~m}, \quad \tau=4 \mathrm{~ms} .
$$

The reference signal thus has sufficient SNR in this configuration to satisfy the $2^{\circ}$ phase noise requirement for a bistatic baseline value of up to $\sim 8000 \mathrm{~m}$ at 4-ms chirp length.

For the scene signal contribution $\sigma_{\phi \text {,scene, }}$, the main differentiating factor between the primary $(\mathrm{P})$ and secondary $(\mathrm{S})$ devices is the reduction of receiver antenna gain. This $12-\mathrm{dB}$ reduction directly translates to reduction of scene SNR for the secondary device and will cause the standard deviation of interferogram phase noise (and the corresponding distance measurement precision) to degrade [48]

$$
\frac{\sigma_{\phi, \text { scene }, \mathrm{S}}}{\sigma_{\phi, \text { scene } \mathrm{P}}}=\frac{1}{\sqrt{10^{-1.2}}} \approx 4 \text {. }
$$

\footnotetext{
${ }^{1}$ The noise floor of the receiver $I_{\text {noise }}$ can be determined by measuring the intensity of the noise within the secondary receiver's SLC dataset, acquired while the primary transmitter is turned off.
} 


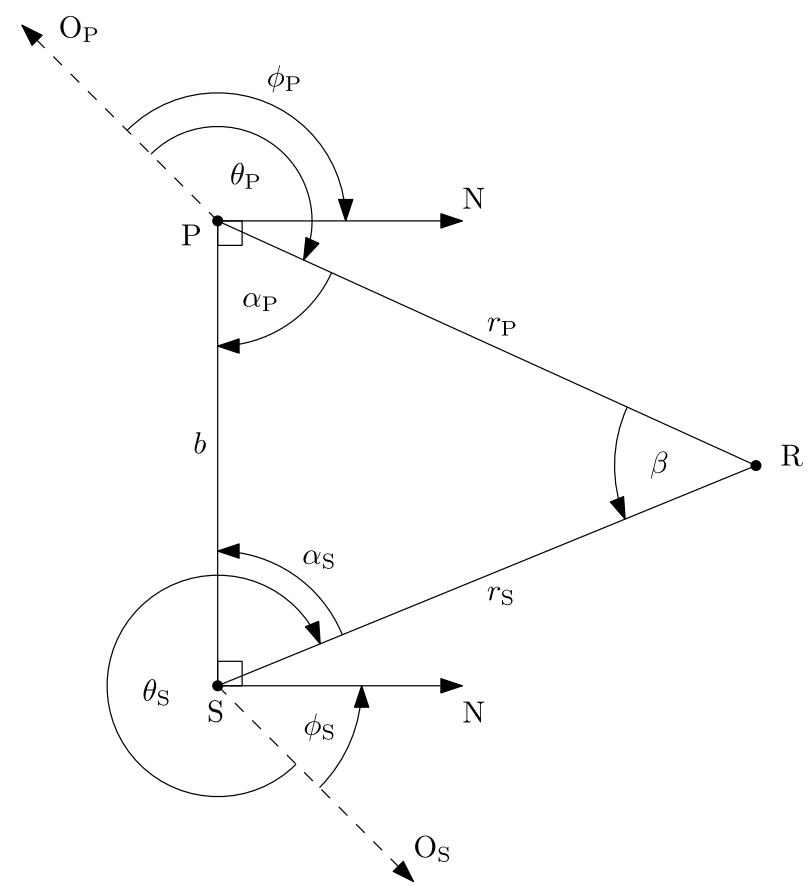

Fig. 5. Bistatic north coordinate system derivation procedure. For all angles, a positive angle value represents a clockwise rotation from the direction marked by the start of the arrow, to the direction marked by the endpoint of the arrow. If the arrow points counterclockwise, the corresponding angle's value is negative. The initial directions of zero-azimuth coordinates $\mathrm{O}_{P}$ and $\mathrm{O}_{S}$ are dependent on particular orientation of the devices' tripods. The objective is then to establish a relation between these directions and the north direction $\mathrm{N}$ of the bistatic coordinate system. This is achieved by using a reference point $\mathrm{P}$ within the scene. Positions of devices $\mathrm{P}$ and $\mathrm{S}$, as well as reference scene point $\mathrm{P}$ are determined by GPS measurements. From their coordinates, angles $\alpha_{\mathrm{P}}$ and $\alpha_{\mathrm{S}}$ are calculated. Monostatic acquisitions of point $\mathrm{P}$ are then performed by both devices, acquiring the value of P's azimuth coordinates $\theta_{\mathrm{P}}$ and $\theta_{\mathrm{S}}$. From this, the azimuth direction of bistatic north can be determined, as $\phi_{\mathrm{P}}=\theta_{\mathrm{P}}+\alpha_{\mathrm{P}}-(\pi / 2)$ and $\phi_{\mathrm{S}}=\theta_{\mathrm{S}}+\alpha_{\mathrm{S}}+(\pi / 2)$.

\section{F. Bistatic Geometry Corrections}

For the purpose of correctly geocoding data within the bistatic acquisition geometry with elliptical iso-range lines, it is important to be able to reference the internal azimuth coordinate of each tower's rotational positioner to the bistatic north-referenced coordinate system shown in Fig. 1 and afterward to real-world geographic coordinates. This can be done by carrying out a monostatic acquisition by each device and referencing a target with known geographic coordinates with its position within the SLC. One narrow-beam transmitter antenna is placed on the secondary device for the purpose of this initial referencing acquisition, the principle of which is shown in Fig. 5.

The bistatic geometry also has specific implications for recovery of the scatterers' range and intensity values.

1) Range: The total signal travel distance $p$ for each scatterer can by recovered by taking the Fourier transform of the deramped corrected signal $s_{\mathrm{d} \text {-corr }}(t)$. In the monostatic case, this travel distance has a trivial connection to range

$$
r_{\mathrm{P}}=r_{\mathrm{S}}=\frac{p}{2}
$$

In the bistatic case, the scatterer's distance to the primary device $r_{\mathrm{P}}$ is calculated as [35]

$$
r_{\mathrm{P}}=\frac{p^{2}-b^{2}}{2\left(p-b \sin \theta_{\mathrm{P}}\right)}
$$

where $b$ is the length of the bistatic baseline, and the scatterer's azimuth coordinate $\theta_{\mathrm{P}}$ is defined by the current rotational position of the narrow-aperture antennas. ${ }^{2}$ After range compression of the acquired signal is performed, the bistatic dataset thus needs to be shifted in range according to (30). This aligns the bistatic SLC with the simultaneously acquired monostatic SLC. ${ }^{3}$ Visualization of this range shift in different parts of the scene is shown in Fig. 6(a).

2) Intensity: It is desired that the intensity of the SLC data is directly proportional to radar brightness $\beta_{0}$. In the monostatic case, this is achieved by scaling each range line of the SLC by $\sqrt{r^{3}}$ to compensate for varying resolution cell size and power spreading loss [40]. In the bistatic case

$$
\beta_{0} \sim \operatorname{Pr}_{\mathrm{P}}^{2} r_{\mathrm{S}}^{2} \frac{\cos ^{2} \beta / 2}{r_{\mathrm{P}}}
$$

where $\beta$ is the bistatic angle, $P$ is the received power, $r_{\mathrm{P}}^{2} r_{\mathrm{S}}^{2}$ is the power spreading term, and $r_{\mathrm{P}}^{-1} \cos ^{2} \beta / 2$ accounts for varying resolution cell area [35], [59]. Thus, the final SLC scaling factor varies in both range and azimuth and is equal to $\left(r_{\mathrm{P}} r_{\mathrm{S}}^{2}\right)^{1 / 2} \cos \beta / 2$. Visualization of the ratio of bistatic to monostatic intensity in different areas of the scene is shown in Fig. 6(b).

\section{G. Antenna Pattern}

The radar equation for a bistatic real-aperture radar system for a single point scatterer is [10]

$$
P_{r}\left(r_{\mathrm{P}}, r_{\mathrm{S}}, \theta_{\mathrm{P}}^{\prime}, \theta_{\mathrm{S}}^{\prime}, \varepsilon_{\mathrm{P}}^{\prime}, \varepsilon_{\mathrm{S}}^{\prime}\right)=\frac{P_{t} G_{t}\left(\theta_{\mathrm{P}}^{\prime}, \varepsilon_{\mathrm{P}}^{\prime}\right) G_{\mathrm{r}}\left(\theta_{\mathrm{S}}^{\prime}, \varepsilon_{\mathrm{S}}^{\prime}\right) \lambda^{2} \sigma}{(4 \pi)^{3} r_{\mathrm{P}}^{2} r_{\mathrm{S}}^{2} L_{s}}
$$

where $P_{t}$ is the transmitted power, $r_{\mathrm{P}}$ and $r_{\mathrm{S}}$ are distances from the primary and secondary devices, respectively, $\sigma$ is the bistatic radar cross section, $L_{s}$ is the system losses, and $G_{t}\left(\theta_{\mathrm{P}}^{\prime}, \varepsilon_{\mathrm{P}}^{\prime}\right), G_{r}\left(\theta_{\mathrm{S}}^{\prime}, \varepsilon_{\mathrm{S}}^{\prime}\right)$ is the antenna gain at the azimuth and elevation offset angle from the respective antenna pointing directions.

For polarimetry, the $G_{t}$ and $G_{r}$ terms become important in case that differently polarized antennas have different offcenter gain patterns. ${ }^{4}$ In that case, intensities of individual polarization channels measured in these off-center pointing directions (but still within the antenna aperture) would be distorted, and as a result, an incorrect polarimetric scattering matrix $S$ would be reconstructed.

The solution is to measure the corresponding antenna gain pattern $G\left(\theta^{\prime}, \varepsilon^{\prime}\right)$ and scale each SLC value by the factor $G\left(\theta^{\prime}, \varepsilon^{\prime}\right)^{-0.5}$. This, of course, comes at a cost of reduced

\footnotetext{
${ }^{2}$ Equation (30) reduces to (29) in the monostatic case $b=0$.

${ }^{3}$ Range resolution will, in general, be worse in the bistatic dataset, especially at bistatic angles $\beta>90^{\circ}$. Length (along range direction) of a range-limited bistatic resolution cell is scaled by a factor of approximately $\cos (\beta / 2)^{-2}$ as opposed to monostatic resolution cell length [35].

${ }^{4}$ Different antenna gains along the central pointing direction are compen-
} sated by polarimetric calibration. 


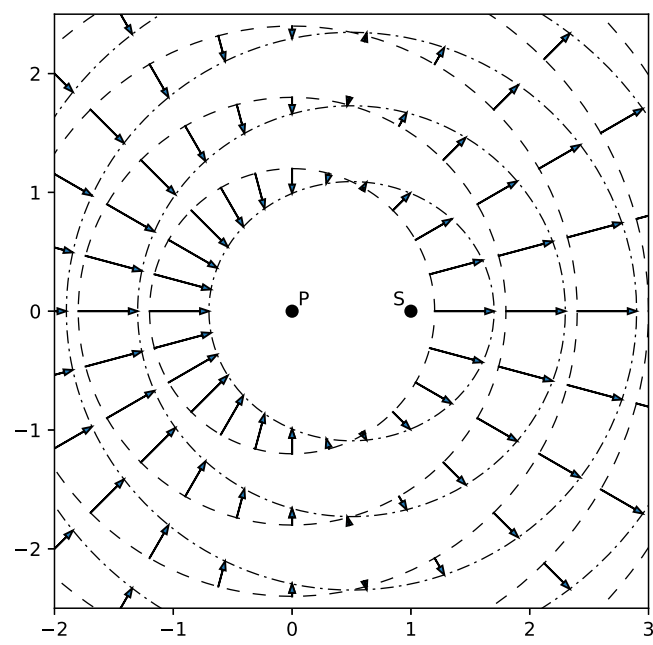

(a)

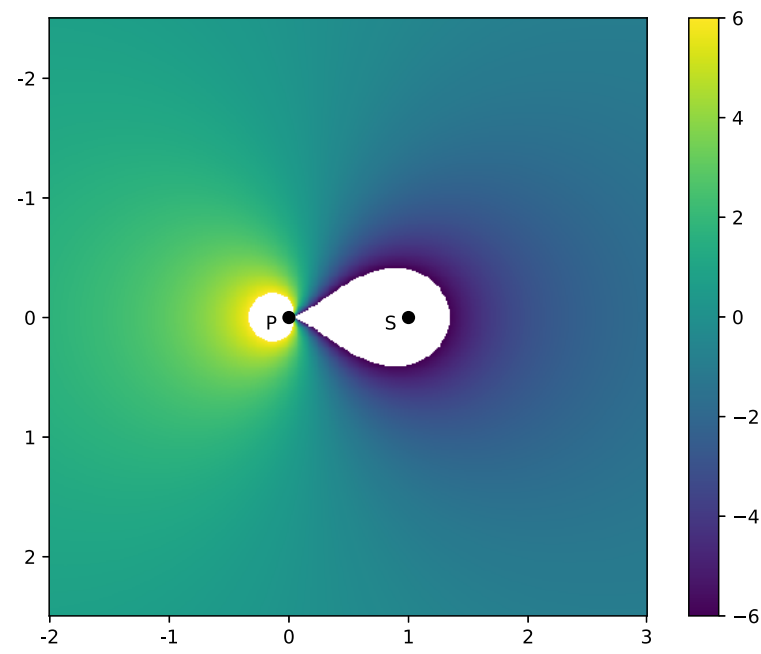

(b)

Fig. 6. Bistatic geometry corrections necessary to align the data acquired by the secondary bistatic receiver with the primary monostatic dataset. The $\mathrm{P}$ and S points mark the location of the primary and secondary device, respectively, and axis labels are in units of bistatic baseline. (a) Correction of observed range according to (30). The start points of the arrows (and the dashed lines) mark the perceived location if monostatic range equation (29) were used, whereas the end points of the arrows (and the corresponding dashed-dotted lines) mark the real location of the target. Targets near the primary device appear further away than they actually are, while targets near the secondary receiver appear closer; (b) Correction of intensity due to varying resolution cell area according to (31). The shown value is the difference in $\mathrm{dB}$ between real $\beta_{0}$ for the bistatic receiver, and $\beta_{0}$ observed using monostatic intensity scaling. An uncorrected bistatic dataset would overestimate $\beta_{0}$ of targets in the vicinity of the secondary receiver while underestimating $\beta_{0}$ of targets close to the primary transmitter. The difference vanishes for large range distances.
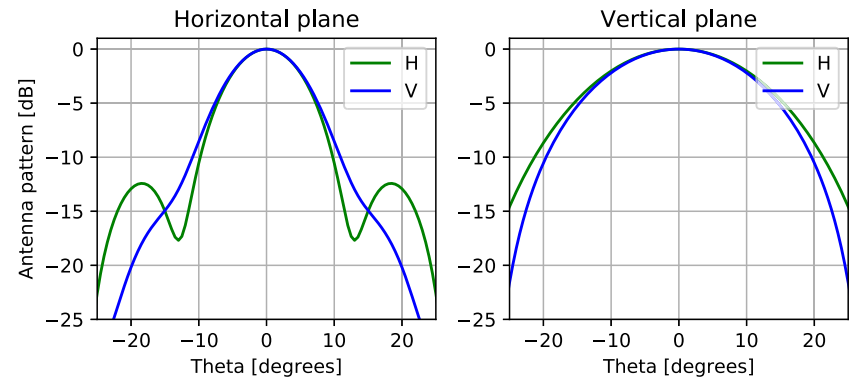

Fig. 7. Calculated co-polarized gain patterns in the horizontal and vertical measurement planes for bistatic Ku-band horn antennas employed by KAPRI.

SNR, so a minimal gain threshold has to be specified, and SLC pixels for which the gain is below this threshold must be discarded.

However, this step is not necessary for the polarimetric analysis, in case the antennas have equal patterns within the cutoff angle, which is the case for the KAPRI radar. Fig. 7 shows the calculated antenna patterns for the $\mathrm{H}$ - and V-polarized horn antennas of the secondary bistatic receiver.

The fact that only the intersection of the two devices' antenna patterns can be imaged has the following implications for bistatic acquisitions.

1) Area coverage of a bistatic acquisition is always lower than in the monostatic case, since while a monostatic acquisition only requires line of sight from one device, a bistatic acquisition requires direct line of sight from every observed point to both transmitter and receiver.

2) The need for mosaicking of multiple acquisitions increases the time and data volume necessary to acquire a complete dataset by a factor of $n \approx \Omega / \delta_{\mathrm{S}}^{\theta}$, where $\Omega$ is the desired total azimuth coverage of the secondary device and $\delta_{\mathrm{S}}^{\theta}$ is the azimuth beamwidth of the secondary device's antennas.

\section{H. Polarimetric Calibration Target}

For the purposes of polarimetric calibration of bistatic KAPRI, an ideal calibration target would have the following properties:

1) a high and stable RCS;

2) capable of returning signal in the desired direction under a variety of bistatic angles;

3) low size and weight for portability in natural environments;

4) ease of alignment under field conditions;

5) capability of calibrating both co- and cross-polarized channels while maintaining identical phase center position for all of these channels.

The first requirement disqualifies targets such as a spherewide aperture of the receiver antennas already causes a reduction in SNR and resolution, and thus, a sphere would have too low of a radar cross section compared to clutter within the same range cell. Other passive targets such as a modified dihedral proposed in [41] would likely have sufficient RCS but fail requirements $2-4$ since they require precise alignment relative to position of the radar devices. For these reasons, an active calibration device was selected, similar to other bistatic campaigns [5], [45], [60].

Our proposed design Variable-Signature Polarimetric Active Radar Calibrator (VSPARC) employs two horn antennas with a Ku-band amplifier in-between..$^{5}$ This ensures sufficient RCS

\footnotetext{
${ }^{5}$ An example of a similar approach can be found in [45] where such a device is used for RCS calibration, as well as [4] where the device was used as a transponder in order to create a bistatic baseline.
} 


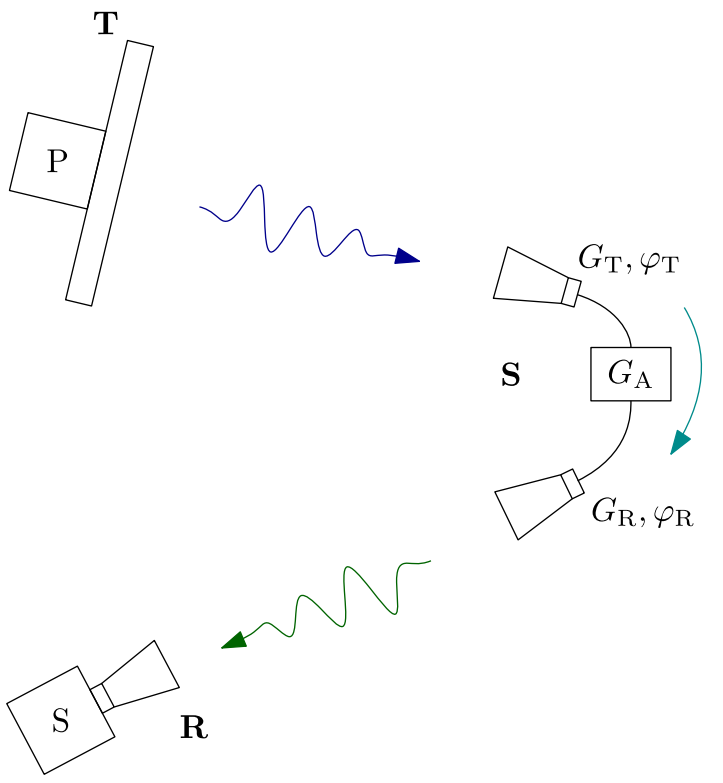

Fig. 8. Schematic of the VSPARC calibration setup. The signal transmitted by the primary device is captured, amplified, and retransmitted by the calibration device toward the secondary. Polarization of the signal is affected by rotation angles $\varphi_{T}$ and $\varphi_{R}$ of the calibrator's antennas.

for calibration even in presence of background clutter and low weight. Directional flexibility and ease of alignment are provided by custom-made mounting adapters for the antennasa manually operated camera mount is used for pointing the antennas in the direction toward the transmitting and receiving radars. Afterward, polarization of the antennas can be adjusted by physical rotation around their line of sight, provided by a rotation stage. The mounting adapter is designed in a way such that the phase center of the antenna lies on the central axis of the rotation stage - changing the polarization of the antenna can be thus done without introducing any additional mechanical phase offsets. A schematic of the device is shown in Fig. 8, and a prototype of the device deployed in the field is shown in Fig. 9.

For both the antenna pointed at the transmitter and the antenna pointed at the receiver, the rotation angle around the pointing axis of the antenna $\left(\varphi_{T}\right.$ and $\varphi_{R}$, respectively) affects the resulting scattering matrix of the calibration target. Sensitivity of the antennas to horizontally and vertically polarized waves can be characterized by a two-component vector

$$
\begin{aligned}
& \mathbf{t}=\sqrt{G_{T}} e^{j \phi_{\mathrm{abs}, \mathrm{T}}\left(\varphi_{T}\right)}\left[\begin{array}{c}
\sin \varphi_{T} \\
\cos \varphi_{T}
\end{array}\right] \\
& \mathbf{r}=\sqrt{G_{R}} e^{j \phi_{\mathrm{abs}, \mathrm{R}}\left(\varphi_{R}\right)}\left[\begin{array}{l}
\sin \varphi_{R} \\
\cos \varphi_{R}
\end{array}\right]
\end{aligned}
$$

where $G_{T}$ and $G_{R}$ are the gain of the respective antenna and $\phi_{\text {abs }}$ describes any phase offsets introduced due to change of the antenna's rotational position $\varphi .^{6}$

The scattering matrix is then computed as the outer product of these two vectors, accounting for line losses $L$ and

\footnotetext{
${ }^{6}$ While the rotational mounts of the antennas were designed to minimize the shifting of the phase center's position during rotation, at Ku-band wavelengths, even a positional shift on the order of $\sim 1 \mathrm{~mm}$ causes a noticeable phase shift.
}

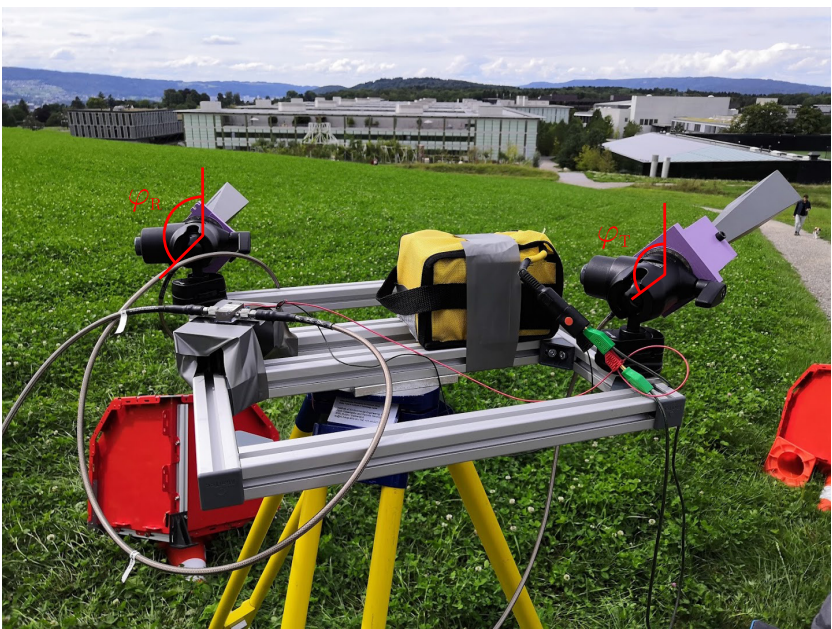

Fig. 9. VSPARC prototype deployed in the field. Antennas are pointed at KAPRI devices and customized 3-D printed adapters enable rotation of antennas around their pointing axis without significantly affecting the position of the phase center. $\varphi_{T}$ and $\varphi_{R}$ quantify the rotation angle of the corresponding antenna around its pointing axis. A zero value of these angles signifies that the corresponding antenna is in the vertically polarized orientation.

amplifier gain $G_{A}$

$$
\mathbf{S}_{\mathrm{cal}}=\sqrt{G_{A} L} \mathbf{r} \otimes \mathbf{t} .
$$

The final scattering matrix of the calibration target then is

$$
\mathbf{S}_{\mathrm{cal}}=e^{j \phi_{\mathrm{abs}}\left(\varphi_{T}, \varphi_{R}\right)} \sqrt{G}\left[\begin{array}{ll}
\sin \varphi_{T} \sin \varphi_{R} & \cos \varphi_{T} \sin \varphi_{R} \\
\sin \varphi_{T} \cos \varphi_{R} & \cos \varphi_{T} \cos \varphi_{R}
\end{array}\right]
$$

where $G=G_{T} L G_{A} G_{R}$ and $\phi_{\mathrm{abs}}\left(\varphi_{T}, \varphi_{R}\right)=\phi_{\mathrm{abs}, T}\left(\varphi_{T}\right)+$ $\phi_{\mathrm{abs}, R}\left(\varphi_{R}\right)$ is the absolute phase delay term, which depends on cable length and precise position of the antennas. The exact values of individual elements of the scattering matrix can thus be altered by changing the rotational position of the antennas.

\section{Polarimetric Calibration Method}

The calibration method is based on the linear distortion matrix model [38], which relates the observed scattering matrix $\mathbf{O}$ to the estimated true target scattering matrix $\mathbf{S}$

$$
\mathbf{O}=\mathbf{R S T}
$$

where $\mathbf{R}$ and $\mathbf{T}$ describe phase offsets, amplitude imbalances, and channel crosstalk for the reception and transmission devices, respectively.

KAPRI by design has very good polarization isolation, and thus, crosstalk is negligible [40]. The horn antennas of the bistatic receiver also provide sufficient polarization isolation, as is verified in Section III-C. The distortion matrices then have the form [40]

$$
\begin{gathered}
\mathbf{R}=\sqrt{A}\left[\begin{array}{cc}
1 & 0 \\
0 & f / g e^{j \phi_{r}}
\end{array}\right] \\
\mathbf{T}=\sqrt{A}\left[\begin{array}{cc}
1 & 0 \\
0 & f g e^{j \phi_{t}}
\end{array}\right] .
\end{gathered}
$$

The four real parameters defined in [40] as $f$ (one-way co-polarized amplitude imbalance relative to the $\mathrm{H}$ polarization), $g$ (one-way cross-polarized amplitude imbalance), 
$\phi_{t}$ (phase offset between polarizations when transmitting), and $\phi_{r}$ (phase offset in reception) can be computed from the covariance matrix $C$

$$
\begin{aligned}
f & =\left(\frac{C_{\mathrm{VVVV}}^{\prime}}{C_{\mathrm{HHHH}}^{\prime}}\right)^{\frac{1}{4}} \\
\phi_{r}+\phi_{t} & =\arg \left(C_{\mathrm{VVHH}}^{\prime}\right) \\
g & =\left\langle\frac{C_{\mathrm{HVHV}}}{C_{\mathrm{VHVH}}}\right\rangle^{\frac{1}{4}} \\
\phi_{t}-\phi_{r} & =\arg \left(\left\langle C_{\mathrm{VVHV}}\right\rangle\right)
\end{aligned}
$$

where the first two equations are evaluated on the response of a corner reflector $C^{\prime}$ and the latter two on the whole scene $C$ (due to the reciprocity principle). This methodhenceforth referred to as the CR (corner reflector) methodwas developed for monostatic KAPRI calibration in [40].

Since, in the generalized bistatic case, neither corner reflectors nor the reciprocity principle can be utilized, a different method has to be developed to compute these four parameters. As a calibration target, we thus use the active calibrator VSPARC described in Section II-H. In order to determine the calibration parameters, we perform acquisitions in five configurations of the calibrator's antennas

$$
\begin{aligned}
& \varphi_{T}=90^{\circ}, \quad \varphi_{R}=90^{\circ} \Longrightarrow \mathbf{S}_{\mathrm{cal}}^{\mathrm{HH}}=\sqrt{G} e^{j \phi_{\mathrm{HH}}}\left[\begin{array}{ll}
1 & 0 \\
0 & 0
\end{array}\right] \\
& \varphi_{T}=90^{\circ}, \quad \varphi_{R}=0^{\circ} \Longrightarrow \mathbf{S}_{\mathrm{cal}}^{\mathrm{VH}}=\sqrt{G} e^{j \phi_{\mathrm{VH}}}\left[\begin{array}{ll}
0 & 0 \\
1 & 0
\end{array}\right] \\
& \varphi_{T}=0^{\circ}, \quad \varphi_{R}=90^{\circ} \Longrightarrow \mathbf{S}_{\mathrm{cal}}^{\mathrm{HV}}=\sqrt{G} e^{j \phi_{\mathrm{HV}}}\left[\begin{array}{ll}
0 & 1 \\
0 & 0
\end{array}\right] \\
& \varphi_{T}=0^{\circ}, \quad \varphi_{R}=0^{\circ} \Longrightarrow \mathbf{S}_{\mathrm{cal}}^{\mathrm{VV}}=\sqrt{G} e^{j \phi_{\mathrm{Vv}}}\left[\begin{array}{ll}
0 & 0 \\
0 & 1
\end{array}\right] \\
& \varphi_{T}=45^{\circ}, \quad \varphi_{R}=45^{\circ} \Longrightarrow \mathbf{S}_{\mathrm{cal}}^{\mathrm{XX}}=\frac{\sqrt{G}}{2} e^{j \phi_{\mathrm{XX}}}\left[\begin{array}{ll}
1 & 1 \\
1 & 1
\end{array}\right]
\end{aligned}
$$

where $G$ is the gain of the calibrator, $\phi_{\mathrm{HH}}, \phi_{\mathrm{VH}} \cdots \phi_{\mathrm{XX}}$ are the absolute phase terms for the corresponding configurations, and the upper index denotes the particular configuration of the antennas. $^{7}$

From measurements of the calibration device's observed scattering matrix $\mathbf{K}$

$$
\mathbf{K}=\mathbf{R S}_{\mathrm{cal}} \mathbf{T}
$$

the four calibration parameters can be computed as follows:

$$
\begin{aligned}
& f=\sqrt{\left|\frac{K_{\mathrm{VV}}^{\mathrm{VV}}}{K_{\mathrm{HH}}^{\mathrm{HH}}}\right|} \\
& g=\sqrt{\left|\frac{K_{\mathrm{HV}}^{\mathrm{HV}}}{K_{\mathrm{VH}}^{\mathrm{VH}}}\right|}
\end{aligned}
$$

\footnotetext{
${ }^{7}$ For example, in (39b), the upper index in notation $\mathbf{S}_{\mathrm{cal}}^{\mathrm{VH}}$ indicates that the calibrator's antenna pointed at the receiver was in the V-sensitive orientation $\left(\varphi_{R}=0^{\circ}\right)$, while the antenna pointed at the transmitter was in the H-sensitive orientation $\left(\varphi_{T}=90^{\circ}\right)$. The letter $\mathrm{X}$ in $(39 \mathrm{e})$ denotes equal sensitivity of the antenna to both polarizations $\left(\varphi=45^{\circ}\right)$.
}

$$
\begin{gathered}
\phi_{r}=\arg \left(\frac{K_{\mathrm{VH}}^{\mathrm{XX}}}{K_{\mathrm{HH}}^{\mathrm{XX}}}\right) \\
\phi_{t}=\arg \left(\frac{K_{\mathrm{HV}}^{\mathrm{XX}}}{K_{\mathrm{HH}}^{\mathrm{XX}}}\right)
\end{gathered}
$$

where the upper index denotes the particular configuration of the calibrator as defined in (39) and the lower index denotes a particular element of the matrix $\mathbf{K}$ observed in that configuration. $^{8}$

These coefficients can then be used to recover the true scattering matrix $\mathbf{S}$ for any target with observed scattering matrix $\mathbf{O}$

$$
\begin{aligned}
\mathbf{S} & =\mathbf{R}^{-1} \mathbf{O T}^{-1} \\
& =A\left[\begin{array}{cc}
O_{\mathrm{HH}} & \frac{e^{-j \phi_{t}}}{f g} O_{\mathrm{HV}} \\
\frac{g e^{-j \phi_{r}}}{f} O_{\mathrm{VH}} & \frac{e^{-j\left(\phi_{r}+\phi_{t}\right)}}{f^{2}} O_{\mathrm{VV}}
\end{array}\right] .
\end{aligned}
$$

Quantifying the value of $A$ is only necessary for radiometric measurements. It can be performed by observing a target with a bistatic radar cross section $\sigma$

$$
A=\frac{\sqrt{\sigma_{\mathrm{HH}}}}{r_{\mathrm{P}} r_{\mathrm{S}}} \frac{1}{\left|O_{\mathrm{HH}}\right|}
$$

where the factor $1 /\left(r_{\mathrm{P}} r_{\mathrm{S}}\right)$ accounts for the power spreading loss. The target can be a well-known target such as a sphere or also the calibrator itself if its gain $G$ is precisely known

$$
A=\frac{\sqrt{G}}{r_{\mathrm{P}} r_{\mathrm{S}}} \frac{1}{\left|K_{\mathrm{HH}}^{\mathrm{HH}}\right|} .
$$

For the purpose of radiometric calibration, this estimation of $A$ should be carried out on SLC values on which range cell area compensation as described in Section II-F2 has not been applied - since these targets can be approximated as point targets, their observed radar brightness is only affected by their range distance and not by the size of the range cell they are contained in.

\section{J. Processing Pipeline Summary}

The processing steps necessary in order to align the bistatic dataset acquired by the secondary device with the primary device's monostatic acquired dataset can be summarized as follows:

1) reference signal compensation as per Section II-D;

2) frequency-dependent antenna squint compensation as described in [40];

3) bistatic range and intensity corrections as per Section II-F;

4) azimuth phase ramp correction as described in [40];

5) antenna pattern compensation/cropping as per Section II-G;

6) polarimetric calibration as per Section II-I;

7) mosaicking of multiple acquisitions to achieve larger coverage;

8) geocoding and data analysis.

${ }^{8}$ For example $K_{\mathrm{VV}}^{\mathrm{HV}}$ indicates the value of the VV element of the calibrator's observed scattering matrix, while it was in a configuration with the T antenna in the V-polarized position and the $\mathrm{R}$ antenna in $\mathrm{H}$-polarized position (see Fig. 8). 


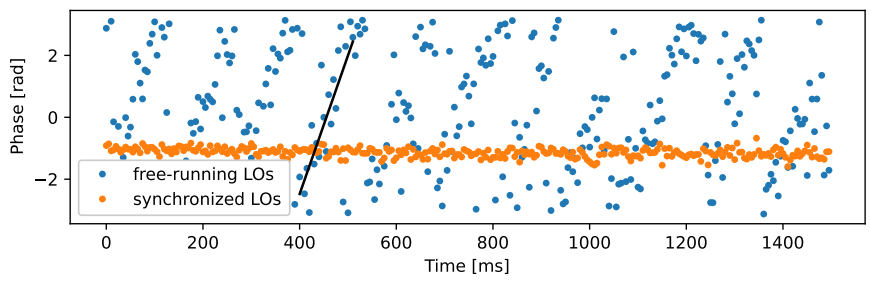

Fig. 10. Measurement of phase drift between the two KAPRI devices. For the orange dataset, both devices were synchronized by a single $100-\mathrm{MHz}$ signal using a cable. In this way, no phase drift between the two devices is observed. The blue dataset corresponds to nominal bistatic operation mode where each device is driven by its own LO, in which case significant phase drift is observed, with an average drift rate of $\sim 2.5^{\circ}$ per millisecond (slope of black line). The signal was transmitted in both cases using a -70 -dB delay line.

\section{DATA AND RESULTS}

\section{A. Phase Drift and Start Time Offset}

Phase drift described in Section II-C5 can be measured by connecting the primary device's transmitter port with the secondary device's receiver port using a delay line-see Fig. 10 .

This measurement can then be used to estimate the clock speed offset between the two devices. Via (13), we can estimate the offset between the device's clock frequencies

$$
\frac{\Delta \tau}{\tau}=-\frac{1}{2 \pi f_{c}^{\prime}} \frac{\mathrm{d} \phi_{\mathrm{d}}}{\mathrm{d} T} .
$$

For the measurement shown in Fig. 10, the average drift rate is estimated to be $\sim 2.5^{\circ} \mathrm{ms}^{-1}$ ( $\mathrm{ms}=$ millisecond), which corresponds to clock frequency offset of

$$
\frac{\Delta \tau}{\tau}=-\frac{2.5^{\circ} \mathrm{ms}^{-1}}{2 \times \pi \times 17.1 \mathrm{GHz}} \approx-4 \times 10^{-10} .
$$

The value of the start time offset $\Delta t$ can also be estimated from the observed range position $p_{\text {ref }}$ and the observed phase $\phi_{\text {ref }}$ of the reference signal peak, as shown in (6) and (11). From (17)

$$
\begin{aligned}
\frac{p_{\text {ref }} \gamma}{c} & =\frac{b \gamma}{c}+\Delta f_{c}-\gamma^{\prime} \Delta t \\
\phi_{\text {ref }} & =2 \pi\left[\frac{b}{\lambda}-\frac{b^{2} \gamma}{2 c^{2}}-f_{c}^{\prime} \Delta t+\frac{\gamma^{\prime}}{2} \Delta t^{2}\right] .
\end{aligned}
$$

Equation (47a) is derived from the frequency of the oscillating term of (17), while (47b) is derived from its phase. From these two expressions, we can obtain two independent estimates of the current value of $\Delta t(T)$

$$
\begin{aligned}
\Delta t_{\text {range }}(T) & =\frac{\gamma\left[b-p_{\text {ref }}(T)\right]}{\gamma^{\prime} c}+\frac{\Delta f_{c}}{\gamma^{\prime}} \\
\Delta t_{\text {phase }}(T) & =-\frac{\phi_{\text {ref }}(T) / 2 \pi-b / \lambda+b^{2} \gamma / 2 c^{2}}{f_{c}^{\prime}}
\end{aligned}
$$

where $T$ represents the slow time, and the second-order phase drift term was neglected in (48b). However, the value of $\left(\Delta f_{c} / \gamma^{\prime}\right)$ in (48a) cannot be isolated given the available data, and the estimate in (48b) is phase-wrapped, and thus, its

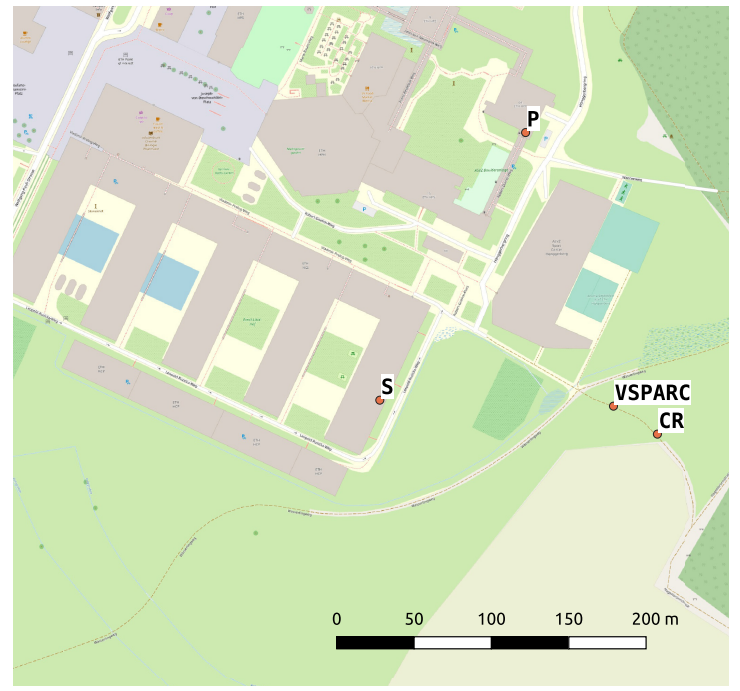

Fig. 11. Synchronization link experimental setup. The bistatic reference signal is transmitted between the primary $(\mathrm{P})$ and secondary $(\mathrm{S})$ devices along a $\sim 200$-m bistatic baseline. Map data $\odot$ openstreetmap.org contributors.

starting absolute value is unknown. For both of these estimates, it is simpler to evaluate $\Delta \hat{t}(T)=\Delta t(T)-\Delta t(0)$

$$
\begin{aligned}
\Delta \hat{t}_{\text {range }}(T) & =\gamma \frac{p_{\text {ref }}(0)-p_{\text {ref }}(T)}{\gamma^{\prime} c} \\
\Delta \hat{t}_{\text {phase }}(T) & =\frac{\phi_{\text {ref }}(0)-\phi_{\text {ref }}(T)}{2 \pi f_{c}^{\prime}}
\end{aligned}
$$

the key being that the absolute offset term in (49a) was subtracted, and the phase information in (49b) can now be unwrapped since the phase history between 0 and $T$ is known. ${ }^{9}$

Fig. 11 shows the geometric configuration for measurement of the reference signal peak range position and phase. Fig. 12 shows the deramped and range-compressed signal containing the reference signal in one of the two channels. Fig. 13 then shows the phase and range coordinate of the reference signal peak and shows the calculated values of $\Delta \hat{t}$ from the reference signal using (49a) and (49b).

\section{B. Calibrator's Scattering Matrix}

We performed a series of acquisitions of VSPARC's polarimetric signature while varying the $\mathrm{R}$ antenna's rotation angle $\varphi_{R}$, while the $\mathrm{T}$ antenna's rotation angle was set to a constant value $\varphi_{T}=45^{\circ}$ (geometric configuration shown in Fig. 14). The scattering matrix of the calibrator then has the form

$$
\mathbf{S}_{\mathrm{cal}}=e^{j \phi_{\mathrm{abs}}\left(\varphi_{R}\right)} \sqrt{\frac{G}{2}}\left[\begin{array}{cc}
\sin \varphi_{R} & \sin \varphi_{R} \\
\cos \varphi_{R} & \cos \varphi_{R}
\end{array}\right] .
$$

The observed signal intensities in individual channels are thus expected to follow a $\cos ^{2}$ trend, with a $90^{\circ}$ offset between the HH-HV and VH-VV pairs. Furthermore, the observed signal phase in each channel should remain approximately constant (with the exception of a $180^{\circ}$ phase flip caused by the changing sign of the respective trigonometric function for each channel) since the antenna mounting adapter was designed

\footnotetext{
${ }^{9}$ Values $\gamma^{\prime}$ and $f_{c}^{\prime}$ can be substituted by their nominal counterparts since the change of their value has orders of magnitude lower effect than the change of value of $p_{\text {ref }}(T)$ and $\phi_{\text {ref }}(T)$, respectively.
} 


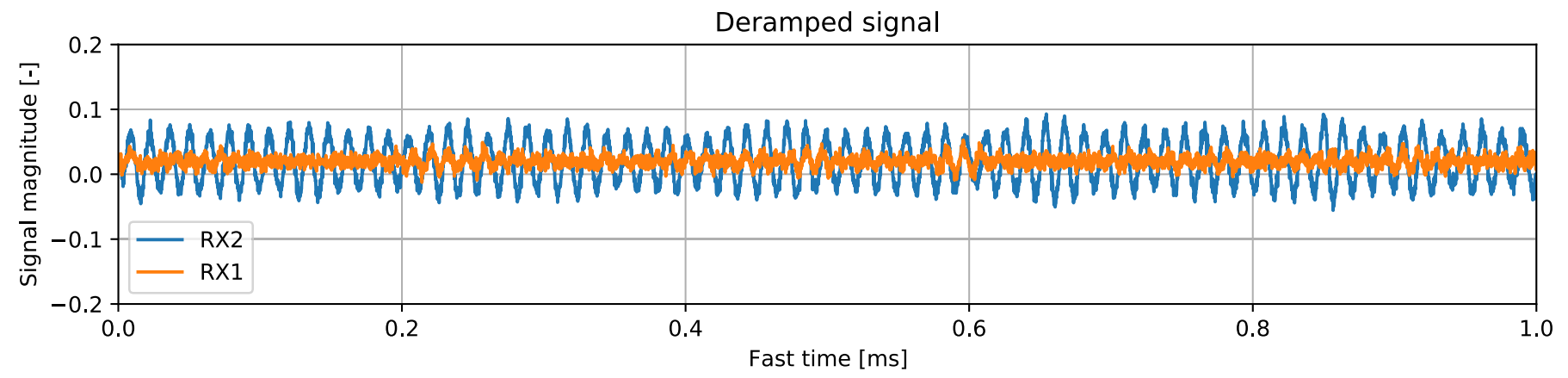

Fig. 12. Deramped and range-compressed signal received by the secondary receiver in the HH polarimetric channel. In channel RX2, strong low-frequency modulation of the deramped signal is caused by the presence of the directly transmitted reference signal (see Figs. 2 and 11 ). The reference signal is visible in the range-compressed data as well as the significant peak at range of $\sim 100 \mathrm{~m}$. The range-compressed signal is scaled in range by a factor of $\left(r^{3}\right)^{1 / 2}$.
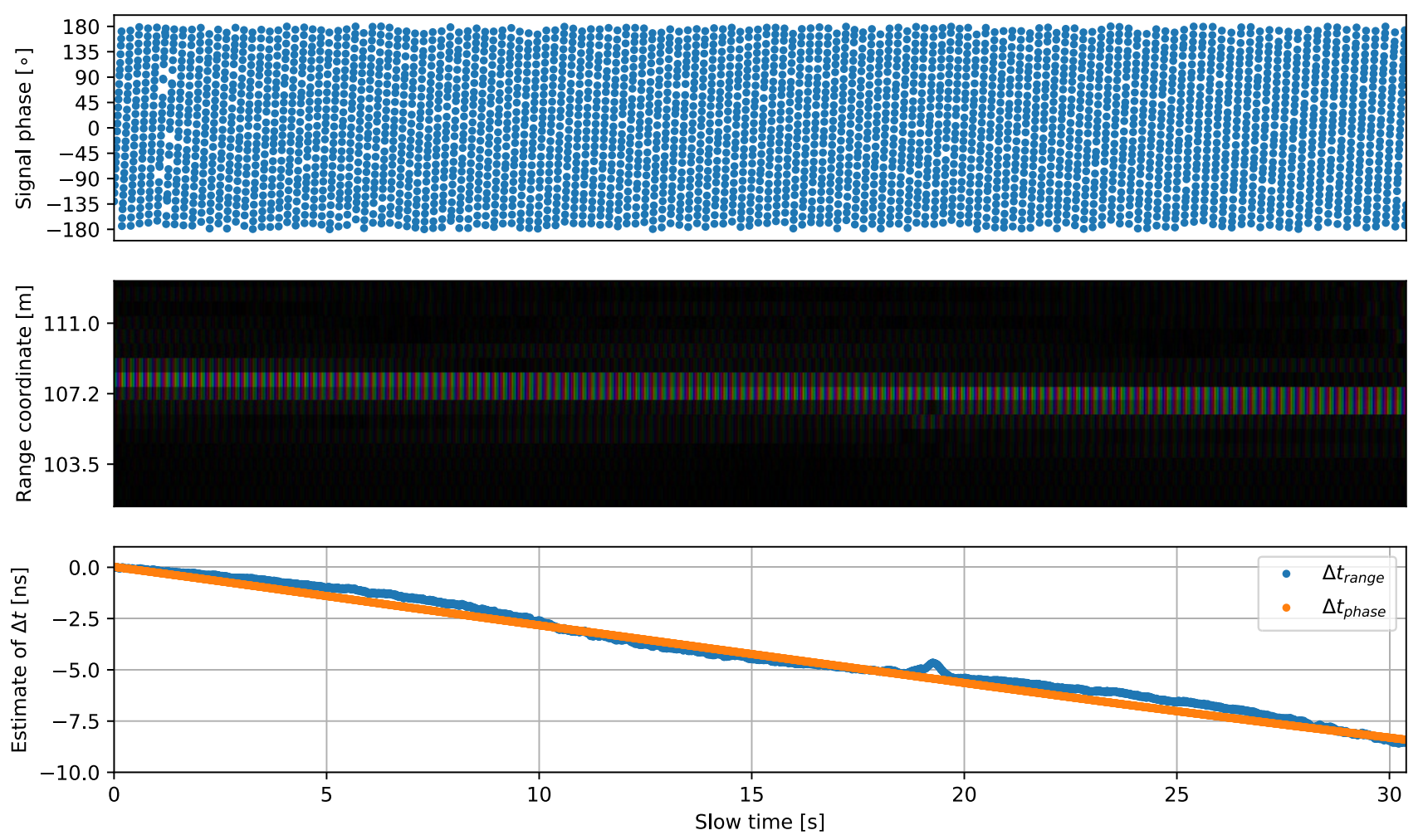

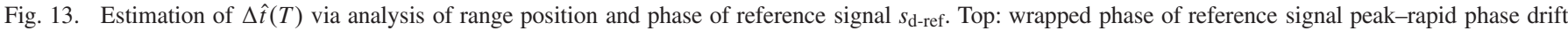
of the reference signal is observed; however, sampling of phase in time is dense enough to allow error-free phase unwrapping. Middle: magnitude (brightness) and phase (color, i.e., hue) of the reference signal within the SLC dataset. A slow drift of the peak toward smaller range coordinate values can be observed. Bottom: estimates of $\Delta \hat{t}(T)$ via (49a) (blue) and (49b) (orange). Small deviation in the blue line in bottom graph at $T \approx 19 \mathrm{~s}$ is caused by a multipath reflection from the scene interfering with the reference chirp. Small deviations of the two estimates are likely caused by imperfect subpixel peak position estimation. Over the course of the acquisition, $\Delta \hat{t}$ accumulates a value of almost $-10 \mathrm{~ns}$. 


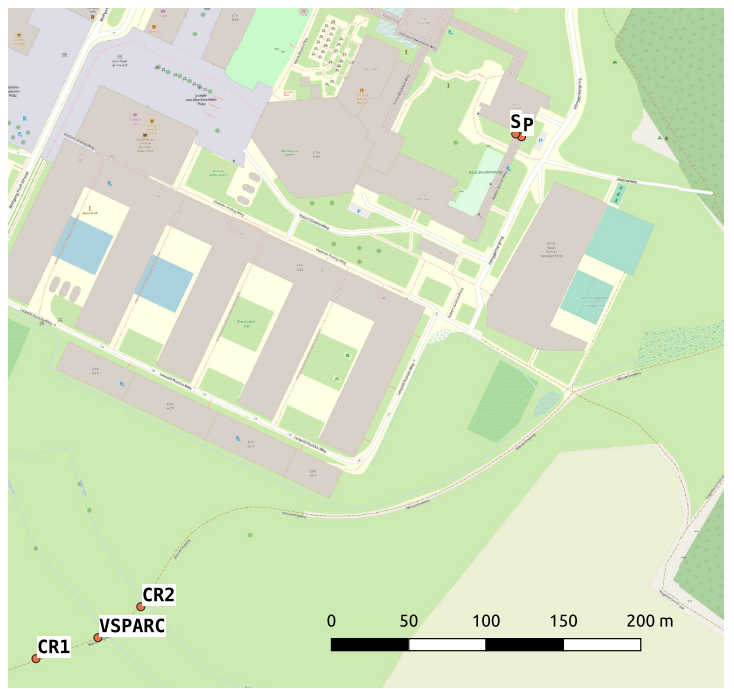

Fig. 14. Polarimetric calibration experimental setup. The primary (P) and secondary (S) devices are placed $\sim 1.5 \mathrm{~m}$ next to each other and thus are both operating with effectively zero bistatic angle. The calibration targets $\mathrm{CR} 1, \mathrm{CR} 2$, and VSPARC are all placed at range distance $\sim 400 \mathrm{~m}$ (however, at different range and azimuth coordinates). This allows the application of both $\mathrm{CR}$ and VSPARC calibration methods for both devices and their comparative validation. Map data (c) openstreetmap.org contributors.
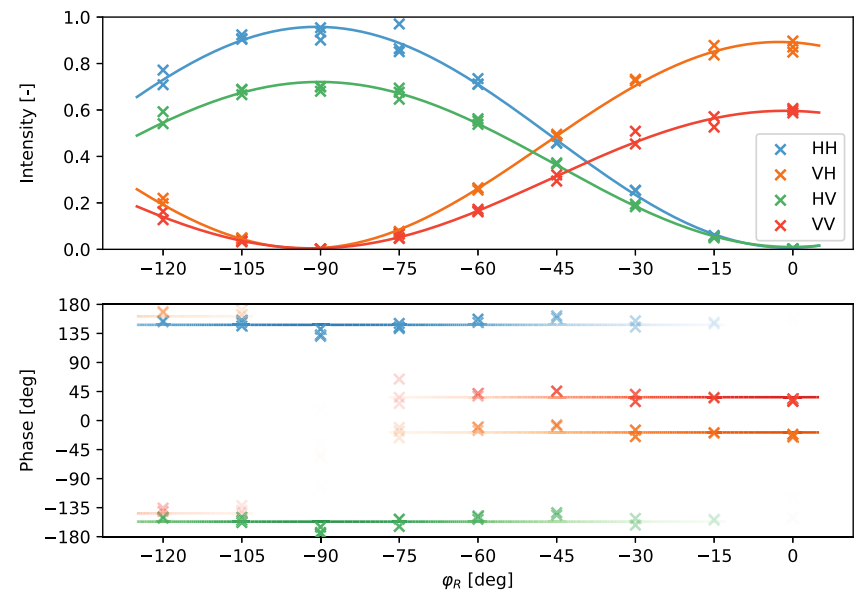

Fig. 15. Measured signal intensity and phase of calibration device VSPARC based on the rotational position of the horn antenna pointed at the receiver $\varphi_{R}$. The color saturation of phase data points and constant fit lines is modulated by signal intensity. The signal intensity follows a $\cos ^{2}$ trend, while the phase for each channel stays constant, just as predicted by (50). A small but noticeable variation of phase around the constant line fits is observed. The predicted $180^{\circ}$ phase flip can be observed in the VH and VV channels by comparing the phase values for antenna rotation angles $\varphi_{R}=-60^{\circ}$ and $\varphi_{R}=-120^{\circ}$ Variations in the amplitudes of the $\cos ^{2}$ curves and variations of observed phase between individual channels are caused by the fact that the dataset is uncalibrated.

to minimize the variation of $\phi_{\mathrm{abs}}\left(\varphi_{R}\right)$ over the course of the rotation. Fig. 15 shows the measured signal intensity and phase of each channel for a range of $\varphi_{R}$ values with a total span of $120^{\circ}$

\section{Polarization Purity}

Table III shows the measured polarization purity of the bistatic receiver, obtained from the scattering response of a corner reflector (with the secondary bistatic receiver placed in the monostatic configuration, i.e., at the same position as the primary transmitter, see Fig. 14).
TABLE III

POLARIZATION PURITY $p$ OF INDIVIDUAL CHANNELS AND THE STANDARD Deviation of the Co-Polar Phase DifFerence $\sigma_{\phi}$,HH-Vv OF THE Primary and Secondary Devices, Measured on Ten ACQUISITIONS OF TWO CORNER REFLECTORS In THE Monostatic Regime

Primary device

\begin{tabular}{|c|c|c|c|}
\hline & $p_{\text {HH-VH }}[\mathrm{dB}]$ & $p_{\mathrm{VV}-\mathrm{HV}}[\mathrm{dB}]$ & $\sigma_{\phi, \mathrm{HH}-\mathrm{Vv}}\left[{ }^{\circ}\right]$ \\
\hline CR1 & $42.8 \pm 1.1$ & $41.8 \pm 0.7$ & 1.2 \\
\hline $\mathrm{CR} 2$ & $33.0 \pm 0.2$ & $33.5 \pm 0.2$ & 2.3 \\
\hline \multicolumn{4}{|c|}{ Secondary device } \\
\hline & $p_{\text {HH-VH }}[\mathrm{dB}]$ & $p_{\mathrm{VV}-\mathrm{HV}}[\mathrm{dB}]$ & $\sigma_{\phi, \mathrm{HH}-\mathrm{VV}}\left[{ }^{\circ}\right]$ \\
\hline CR1 & $29.0 \pm 0.4$ & $37.9 \pm 0.4$ & 1.1 \\
\hline CR2 & $25.3 \pm 0.1$ & $31.5 \pm 0.3$ & 8.1 \\
\hline
\end{tabular}

TABLE IV

RETRIEVED CALIBRATION COEFFICIENTS FOR EACH TARGET AND THE CORRESPONDING METHOD. COEFFICIENTS FOR CORNER REFLECTORS CR1 AND CR2 WERE COMPUTED ACCORDING TO (38) AND FOR CALIBRATION DEVICE VSPARC ACCORDING TO (41). THE Displayed Values are Mean and Standard DeViation OVER ThreE MEASUREMENTS

Primary device

\begin{tabular}{rcccc}
\multicolumn{5}{c}{ Primary device } \\
\hline CR1 & $0.95 \pm 0.01$ & $0.97 \pm 0.01$ & $-100.7 \pm 0.6$ & $12.9 \pm 0.3$ \\
CR2 & $0.95 \pm 0.01$ & $0.98 \pm 0.01$ & $-90.3 \pm 1.0$ & $22.4 \pm 0.7$ \\
VSPARC & $0.92 \pm 0.02$ & $0.99 \pm 0.01$ & $-90.1 \pm 0.8$ & $11.9 \pm 1.2$ \\
\hline \multicolumn{5}{c}{ Secondary device } \\
\hline \multicolumn{5}{c}{$\mathrm{g}$} \\
\hline f $\left[^{\circ}\right]$ & $\phi_{t}\left[^{\circ}\right]$ & $\phi_{r}\left[^{\circ}\right]$ \\
\hline CR2 & $0.96 \pm 0.02$ & $1.02 \pm 0.01$ & $-111.3 \pm 0.5$ & $25.8 \pm 0.7$ \\
VSPARC & $0.99 \pm 0.07$ & $1.03 \pm 0.01$ & $-98.9 \pm 9.5$ & $42.1 \pm 9.4$ \\
\hline
\end{tabular}

\section{Polarimetric Calibration Validation}

The new calibration method presented in Section II-I (VSPARC method) should yield the same results as the original calibration method described in [40] (CR method). The calibration device VSPARC presented in Section II-H can be used for monostatic calibration by pointing both antennas in the same direction.

Table IV shows the retrieved calibration coefficients for the primary and secondary device in a monostatic configuration (i.e., both devices placed next to each other, see Fig. 14) for two corner reflectors CR1 and CR2 employing the CR method [see (38)] and the active calibration device employing the VSPARC method [see (41)].

Table V compares the recovered phase offsets of the two methods in a specific configuration where $\phi_{t}-\phi_{r}<-180^{\circ}$, which triggers a phase-wrapping effect that affects the retrieval of phase coefficients by the calibration method described by (38).

\section{E. Bistatic Processing Steps Visualization}

Fig. 16 shows the key processing steps outlined in Section II-J. 


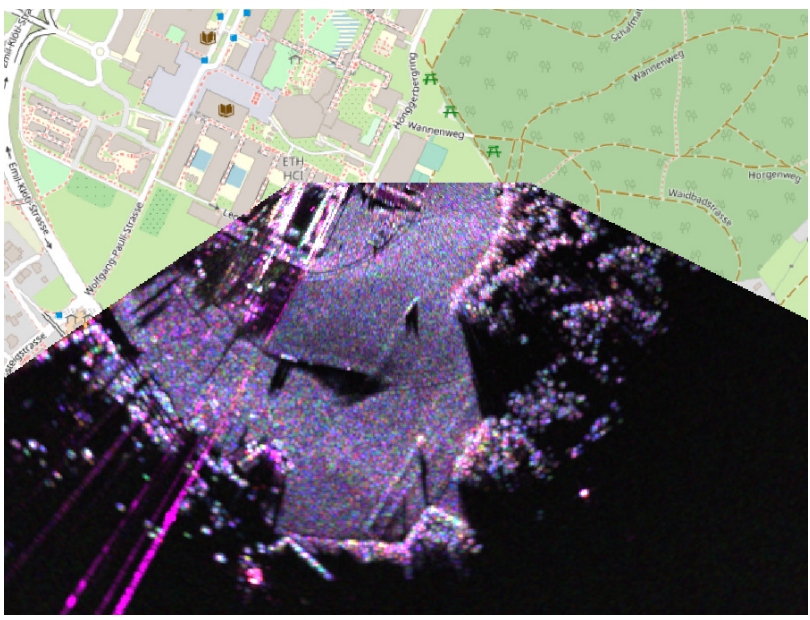

(a)

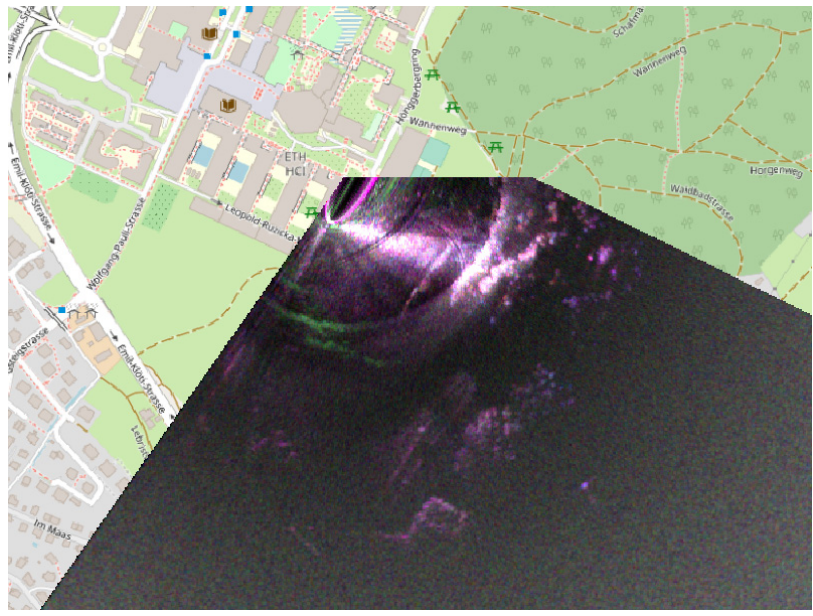

(c)

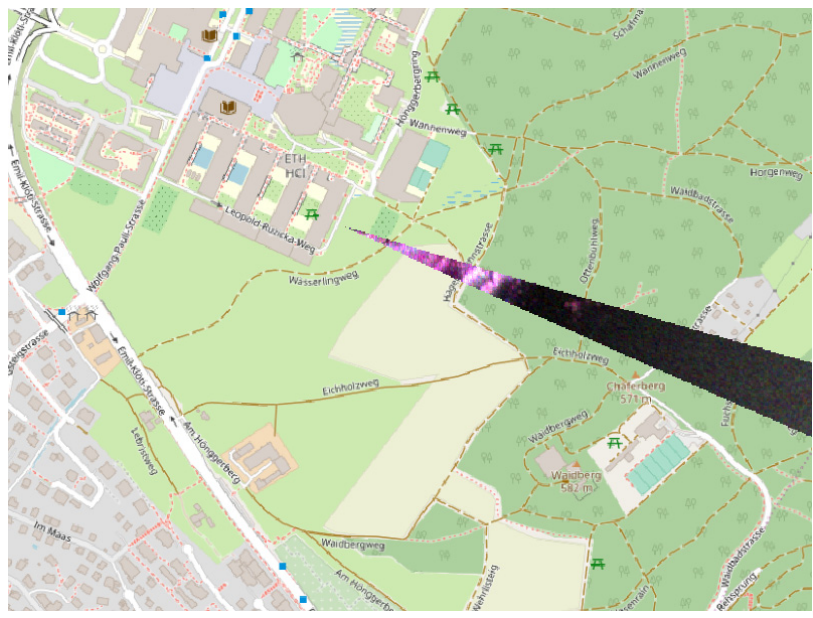

(e)

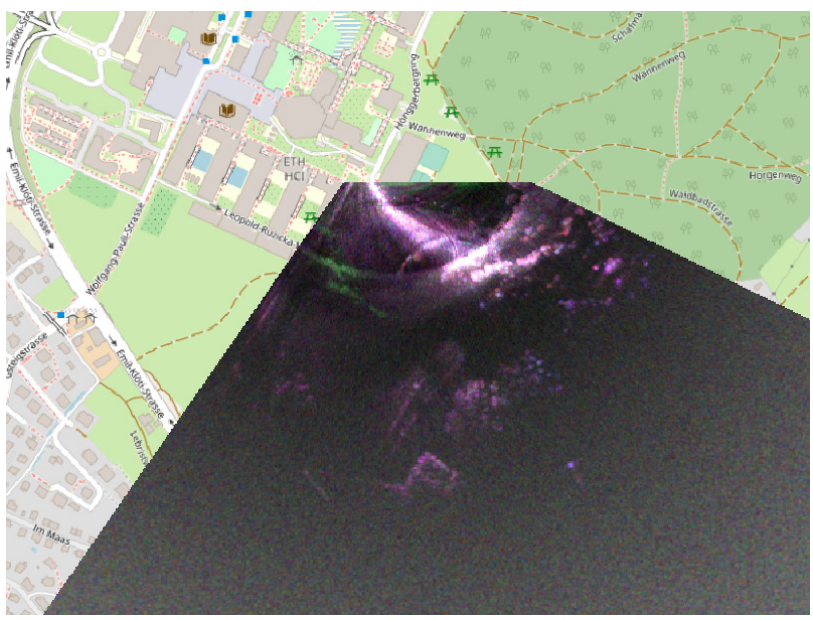

(b)

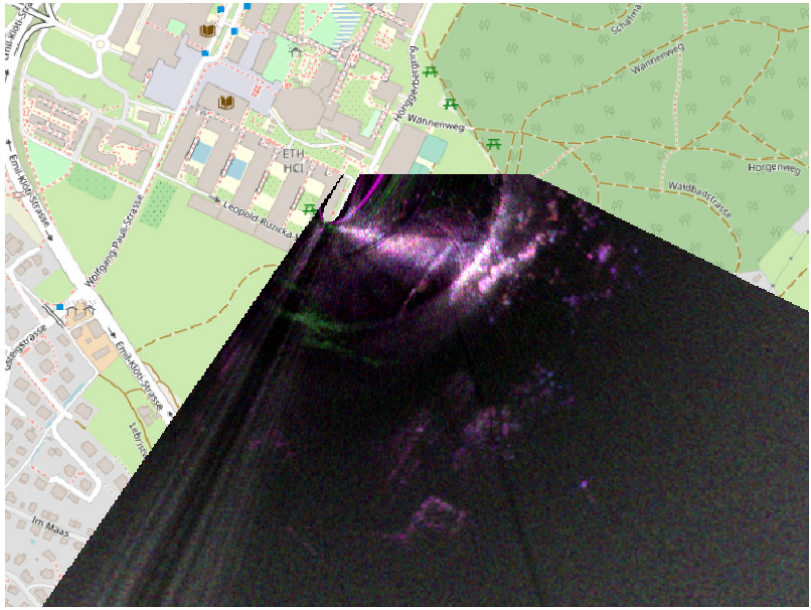

(d)

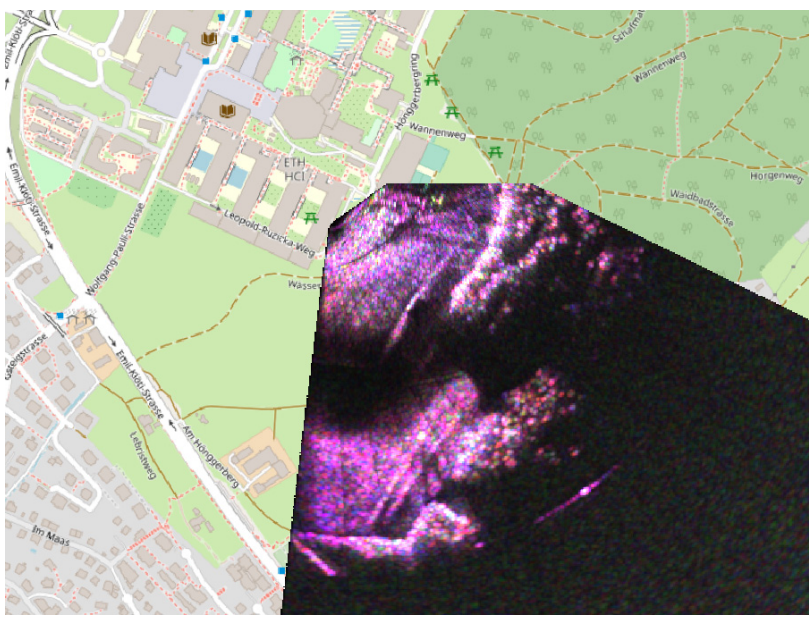

(f)

Fig. 16. Visualization of key processing steps in the bistatic processing pipeline. Data acquired on the ETH Hönggerberg campus. Polarimetric data are presented in the Pauli color basis (R: HH-VV, G: HV, and B: HH+VV). Map data (C) openstreetmap.org contributors. (a) Monostatic image. The primary device was placed on top of a building and thus had very good line of sight over the observed area; (b) Bistatic image acquired by the secondary device and "naively" processed using the monostatic pipeline. Note the much lower coverage, incorrect placement and orientation of the treeline, as well as intensity gradients in both range and azimuth; (c) Range correction per (30). Data are now correctly located, and however, the intensity values are still too high in the vicinity of the receiver; (d) Intensity correction per (31). Intensity gradient in range is now corrected, and however, the azimuth gradient caused by the horn antenna pattern is still well visible; (e) Cropping of off-center data values. Only values that lie up to $\pm 4^{\circ}$ from central azimuth direction are kept (up to $\pm 6^{\circ}$, corresponding to $-3 \mathrm{~dB}$ beamwidth, is possible). Multiple acquisitions are necessary to cover a wider area. Polarimetric calibration is now applied; (f) Mosaicking of multiple bistatic acquisitions with varying horn receiver orientations in order to achieve larger coverage. Only areas with direct line of sight to both primary and secondary devices are imaged. This mosaicking method causes radial "stripes" emanating from the position of the secondary receiver to appear in the image, which is an artifact caused by the modulation of intensity by the receiver antenna pattern. This only affects radiometric information—relative intensities and phases between individual polarimetric channels are preserved. 
TABLE V

Recovered Calibration Phase Offsets for the SeCondary Device In A SPECific Situation Where $\phi_{t}-\phi_{r}<-180^{\circ}$, REsulting in PhaSe WrapPing. COEFFICIENTS FOR CORNER REFLECTORS CR1 AND CR2 Were COMPUTED ACCORDING TO (38) AND FOR CALIBRATION DEVICE VSPARC ACCORDING TO (41). The Displayed Values Are Mean and Standard Deviation Over Three MeasureMENTS. A LARGE DISCREPANCY BETWEEN Recovered Phase Coefficients Between THE Two Methods Is OBSERVED

\begin{tabular}{rcc}
\hline & $\phi_{t}\left[^{\circ}\right]$ & $\phi_{r}\left[^{\circ}\right]$ \\
\hline CR1 & $65.7 \pm 0.4$ & $-81.1 \pm 0.1$ \\
CR2 & $86.3 \pm 9.2$ & $-65.4 \pm 9.8$ \\
VSPARC & $-101.8 \pm 0.6$ & $90.2 \pm 2.0$ \\
\hline
\end{tabular}

\section{DISCUSSION}

\section{A. Geometric Limitations}

Due to KAPRI's synchronization link and the fact that it consists of two synchronized standalone devices, it is possible to perform acquisitions in a wide range of bistatic geometries, the main limiting factor being the local topography. The requirements for a successful acquisition are as follows.

1) There has to exist a direct line of sight between the two devices and between each of the devices and the observed scene-this is observed in Fig. 16, where a "blind spot" is apparent in the center of Fig. 16(f) compared to Fig. 16(a).

2) Distances of the two devices to the observed scene should be within the same order of magnitude, in order to limit the magnitude of the range and intensity shift effects visualized in Fig. 6 .

3) The bistatic angle $\beta$ should not exceed $120^{\circ}$, in order to prevent leakage of the synchronization signal into the observed scene and excessive degradation of range resolution.

\section{B. Signal Model and Phase Retrieval}

As shown in Fig. 10, a clock drift between the two devices (which can accumulate to a value of several tens of nanoseconds) would have a considerable effect on the recovered signal phase in the bistatic configuration (which in the measurement shown exhibits a phase drift of approximately $2.5^{\circ} \mathrm{ms}^{-1}$, but is not constant between individual acquisitions), and thus must be corrected. The dual-chirp signal model introduced and described in Sections II-B-II-D is used to apply a correction via a transmitted reference chirp, and Fig. 13 shows that the model accurately establishes the relation between clock offset and reference signal phase and range offsets. It can be seen that indeed, the phase and range drift of the reference signal are connected and are dominantly caused by the accumulation of a chirp start time offset $\Delta t$. Furthermore, the measurement quantifies that over the course of a typical acquisition $(10-60 \mathrm{~s})$, the start time offset $\Delta t$ can reach a value of several tens of nanoseconds. The observed drift of the reference peak's range coordinate over the slow time also confirms that uncorrected accumulation of $\Delta t$ would cause incorrect range determination for the reference signal, as well as the observed scene.
The corrected deramped signal $s_{\text {d-corr }}(t)$ can be expressed through (5a), (17a), and (19a) as

$$
\begin{aligned}
s_{\mathrm{d}-\text { corr }}(t) & =s_{\mathrm{d}}(t) s_{\mathrm{d}-\mathrm{ref}}(t)^{*} e^{j 2 \pi \frac{b y}{c} t} \\
& =s_{\mathrm{sr}}(t)^{*} s_{\mathrm{st}}(t) s_{\mathrm{sr}-\mathrm{ref}}(t) s_{\mathrm{st}}(t)^{*} e^{j 2 \pi \frac{b y}{c} t} \\
& =s_{\mathrm{sr}}(t)^{*} s_{\mathrm{sr}-\text { ref }}(t) e^{j 2 \pi \frac{b y}{c} t} .
\end{aligned}
$$

Notably, (51c) shows that $s_{\mathrm{d}-\mathrm{corr}}(t)$ does not directly depend on the particular form of $s_{\text {sr-ref }}(t)$, and thus, the method could be used to correct chirps that have a form different from the specific one described by (4). However, knowledge of the properties of $s_{\text {sr-ref }}(t)$ (especially its frequency spectrum) is still important-especially in the KAPRI configurationsince the bistatic reference signal $s_{\mathrm{d} \text {-ref }}(t)$ is not captured in its own dedicated channel, but is instead superimposed on $s_{\mathrm{d}}(t)$. Its recovery is then performed by windowing in the rangecompressed domain as shown in (20), which requires the frequency spectrum of $s_{\mathrm{d}-\text { ref }}(t)$ to be disjoint from the spectrum of $s_{\mathrm{d}}(t)$. The reference signal $s_{\mathrm{d}-\mathrm{ref}}$ is transmitted one way along the bistatic baseline, as opposed to the two-way path of the scene signal $s_{\mathrm{d}}$. This means that the intensity of $s_{\mathrm{d}-\mathrm{ref}}$ is inversely proportional to the square of the length scale of the scene, as opposed to the inverse third power dependence of intensity of $s_{\mathrm{d}}$ (assuming scattering from a surface of distributed targets). Furthermore, in a typical acquisition geometry, the scene antenna beams are pointed away from the radar devices themselves (i.e., the radars do not point the antennas at each other) and thus signal from areas where $p \approx b$ is suppressed, which results in a negligible clutter contribution in the range cell of the reference signal. These two effects result in very good reference signal recovery from the superimposed signal. This is shown in Fig. 12 where the reference signal can be seen as a very clear modulation of the deramped signal in channel $\mathrm{RX} 2$, resulting in a sharp peak in the range-compressed signal at perceived range coordinate $\sim 100 \mathrm{~m}$ (i.e., half of the physical bistatic baseline).

Just like in monostatic configuration, phase retrieval accuracy is influenced by the scene SNR, which is reduced in the bistatic case due to the use of lower gain antennas, with an expected phase noise standard deviation increase by a factor of 4. Table III shows the polarization purity and standard deviation of the co-polar phase difference of two corner reflectors CR1 and CR2. The lower values of polarization purity and higher phase noise of CR2 for the primary device suggest that there was a higher presence of clutter at CR2's position. For the secondary device, the effect of clutter is exacerbated by the lower gain (and higher beamwidth) of the receiver antennas and results in a $\sim 4 \times$ increase of the phase standard deviation, in agreement with (28). The equal values of phase noise for CR1 can be interpreted in a way that there was a very low amount of background clutter present in the scene near CR1, and thus, the SNR for both primary and secondary devices was sufficiently high so that the majority of the observed variation of phase is not caused by signal noise, but by other effects such as atmospheric variations, antenna vibrations, and other temporal phenomena that equally affect both the primary and secondary datasets. 


\section{Polarimetric Calibration}

Table III shows that overall polarization purity of $\sim 30 \mathrm{~dB}$ for the bistatic secondary receiver is lower than the $\sim 40-\mathrm{dB}$ value for the primary device [40]. This is caused by the use of horn antennas for the secondary receiver since these antennas have a smaller form factor and only a single mounting point, which makes them more sensitive to small mounting orientation variations. The bistatic configuration nevertheless still shows very good polarization isolation and is suitable for application of the polarimetric calibration method [38].

Table IV compares the monostatic calibration method (CR), which uses corner reflectors and applies the reciprocity principle, to the novel calibration method by an active calibrator (VSPARC). Both methods provide comparable estimates of the calibration parameters $f, g, \phi_{r}$, and $\phi_{t}$. Amplitude imbalance values close to 1 indicate that there is only a small variation in the real gain of the devices' antennas between individual channels. Coefficients reported in [40, Table V] across multiple corner reflectors exhibit similar variation as the results in Table IV, and within Table IV for both the primary and the secondary device, the VSPARC results are well aligned with the CR1 and CR2 results. It is not expected that the values of parameters $f$ and $\phi_{r}+\phi_{t}$ given in [40] and the primary device parameters of Table IV should be numerically equal since the coefficients reported in [40] are residuals computed on already calibrated data. Furthermore, any component changes to the device configuration such as the addition of the directional coupler also affect the parameter values.

Since both the primary and the secondary device use the same pair of transmitting antennas, the estimate of phase offset at transmission $\phi_{t}$ should have equal value for both devices, which is confirmed in the data with all estimates close to the value of $-90^{\circ}$. The phase offset at reception $\phi_{r}$ is unrelated between the primary and the secondary device; however, for both devices, there is a good match between the estimate of the new and the old method. The data indicate that the accuracy of $20^{\circ}$ can be achieved for the phase calibration in regular operation using the active calibration device.

The CR method [40] derives the phase offsets $\phi_{r}$ and $\phi_{t}$ from (38b) and (38d), where their sum and their difference are estimated from the phases of particular elements of corresponding covariance matrices. However, in case either the sum or the difference of the two parameters exceeds the phasewrapping threshold of $\pm 180^{\circ}$, one of the covariance matrix elements on the right-hand side of (38b) and (38d) will be phase-wrapped, and as a result, the estimates of parameters $\phi_{r}$ and $\phi_{t}$ will be shifted by $180^{\circ}$. Table $\mathrm{V}$ showcases this situation, where the VSPARC observations suggest that the difference $\phi_{t}-\phi_{r}$ is phase-wrapped and CR1 and CR2 observations are phase-shifted from the VSPARC observations by $180^{\circ}$ (within the margin of error). While it is trivial to recover the true coefficients from the CR1 and CR2 estimates, the CR method provides no way to detect the situation when the phase-wrapping criterion is triggered and the $180^{\circ}$ correction is necessary. The VSPARC method provides direct access to $\phi_{r}$ and $\phi_{t}$ from (41c) to (41d) and thus is not affected by this phase-wrapping phenomenon.
Theoretically, it would also be possible to derive the phase offsets $\phi_{r}$ and $\phi_{t}$ from measurements of the VSPARC device in the three configurations described by (39a), (39b), and (39c), thus eliminating the need for measurement in the XX configuration described by (39e). However, the XX configuration has the benefit that both offsets are calculated from a single acquisition in one configuration. This eliminates any errors caused by atmospheric phase screen variations, temporal drift, or any mechanical phase offsets that would be introduced while adjusting the calibration device's configuration-these mechanical offsets can be observed as small deviations of the signal phase from the constant fit line in Fig. 15 and are an expression of the $e^{j \phi_{\mathrm{abs}}\left(\varphi_{\mathrm{T}}, \varphi_{\mathrm{R}}\right)}$ term of (35). Conversely, for the estimation of amplitude imbalances $f$ and $g$, employment of only the XX configuration would be very sensitive to small deviations of the antenna rotation angles $\varphi_{\mathrm{T}}, \varphi_{\mathrm{R}}$ from the optimal $45^{\circ}$ value since (as (35) predicts), at this value, even a small deviation causes a considerable change in signal amplitude (as can be seen in Fig. 15, where the intensity curves have the steepest slope at angle $\varphi_{R}=45^{\circ}$ ). By deriving the amplitude imbalance estimates $f, g$ from measurements where the antenna rotation angles have value $0^{\circ}$ or $90^{\circ}$ (i.e., the stationary points of the intensity curves), this sensitivity to small variations of angles $\varphi_{T}$ and $\varphi_{R}$ is mitigated.

As opposed to the CR method's employment of a passive corner reflector, the VSPARC method necessitates active operation of the device during calibration. The active calibrator design, however, enables polarimetric calibration in configurations with nonzero bistatic angle, which is necessary for bistatic KAPRI operation.

\section{Applications}

Full-polarimetric-interferometric capabilities of bistatic KAPRI, combined with its flexibility in temporal sampling rates and coverage periods, open up possibilities for investigations in several areas:

1) Three-Dimensional Displacement Monitoring: Bistatic interferometric capabilities of KAPRI allow reconstruction of 3-D displacement vector fields [3], [61], with high temporal sampling frequency for monitoring of phenomena that occur both on timescales faster than the ones that can be monitored using satellite-based SAR and longer timescales than those that can be reasonably monitored using airborne instruments. The reduced SNR and wider antenna pattern of the secondary device will result in a reduction of precision by an estimated factor of 4; however, given the excellent resolution and sensitivity of the monostatic GPRI and Ku-band wavelength, the bistatic add-on can still provide submillimeter sensitivity $(0.25-\mathrm{mm}$ path length measurement standard deviation at $10^{\circ}$ phase noise standard deviation [48]), as well as providing opportunities for enhancement of atmospheric phase screen compensation models that affect terrestrial radar observations [47].

2) Top-Layer Vegetation Monitoring: Past research has demonstrated sensitivity of Ku-band radar to biophysical properties of top-layer vegetation [62]. Investigation of bistatic polarimetric radar signatures of vegetated land over the growth 
cycle is a possible pathway toward improvement of methods of surface and biophysical parameter retrieval [7]-[9], [63].

3) Snow and Ice: Certain scattering phenomena occurring in snow and ice layers are only detectable in the bistatic regime, such as the coherent backscatter opposition effect [64], [65]. As a portable real-aperture fully polarimetric bistatic system, KAPRI is a suitable tool for investigation of this and similar phenomena.

\section{CONCLUSion}

In this article, we presented the calibration and the longbaseline bistatic acquisition configuration of KAPRI. By transmitting a synchronization signal directly between the two devices, we are able to compensate for oscillator drift effects without compromising the phase retrieval accuracy of the secondary device, due to the high SNR of the bistatic reference signal. The phase retrieval accuracy of the secondary receiver is then mainly affected by reduced SNR due to lower gain of the receiver antennas, which results in a $4 \times$ increase in standard deviation of phase noise.

For polarimetric calibration, we developed a simple active transponder design VSPARC, which allows calibration of all polarimetric channels based on five measurements of its scattering matrix in specified configurations. This design is well-suited for field operations and can be used for bistatic as well as monostatic calibration, in which case the returned coefficients match the coefficients retrieved from the previously used monostatic method within $20^{\circ}$ and $5 \%$ for phase and amplitude imbalances, respectively. We suggest that the VSPARC device and the associated calibration method can also be applied to calibrate other bistatic radar systems with good polarization isolation.

A fully calibrated KAPRI system can be used to investigate the suitability of bistatic Ku-band radar for probing of a variety of natural phenomena, such as 3-D land movements, vegetation growth, and structural changes in snow and ice.

\section{ACKNOWLEDGMENT}

The authors would like to thank Raphael Wicki, Tingting $\mathrm{Li}$, Yuta Izumi, and Michael Arnold for their assistance with field campaigns and Simone Baffelli for fruitful discussions about bistatic capabilities of KAPRI.

\section{REFERENCES}

[1] J.-S. Lee and E. Pottier, Polarimetric Radar Imaging: From Basics to Applications. Boca Raton, FL, USA: CRC Press, 2009.

[2] Etienne Everaere, "Polarimetry in bistatic configuration for ultra high frequency radar measurements on forest environment," Ph.D. dissertation, Optics, École Polytechnique, 2015. [Online]. Available: https://hal.archives-ouvertes.fr/tel-01199522

[3] A. Moreira et al., "Tandem-L: A highly innovative bistatic SAR mission for global observation of dynamic processes on the earth's surface," IEEE Geosci. Remote Sens. Mag., vol. 3, no. 2, pp. 8-23, Jun. 2015.

[4] M. Pieraccini and L. Miccinesi, "Bistatic GBSAR for detecting target elevation," in Proc. IEEE Int. Conf. Microw., Antennas, Commun. Electron. Syst. (COMCAS), Nov. 2017, pp. 1-4.
[5] P. Dubois-Fernandez et al., "ONERA-DLR bistatic SAR campaign: Planning, data acquisition, and first analysis of bistatic scattering behaviour of natural and urban targets," IEE Proc.-Radar, Sonar Navigat., vol. 153, no. 3, pp. 214-223, Jul. 2006.

[6] W. A. Al-Ashwal et al., "Measurements of bistatic radar sea clutter," in Proc. IEEE RadarCon (RADAR), May 2011, pp. 217-221. [Online]. Available: http://ieeexplore.ieee.org/document/5960531/

[7] L. Villard, I. Hajnsek, P. Borderies, and K. Papathanassiou, "Pol-InSAR simulations in forest bistatic scattering," in Proc. 7th Eur. Conf. Synth. Aperture Radar, Jun. 2008, pp. 3-6.

[8] K. B. Khadhra, T. Boerner, D. Hounam, and M. Chandra, "Surface parameter estimation using bistatic polarimetric X-band measurements," Prog. Electromagn. Res. B, vol. 39, pp. 197-223, 2012. [Online]. Available: http://www.jpier.org/PIERB/pier.php?paper=12020112

[9] E. Erten, J. M. Lopez-Sanchez, O. Yuzugullu, and I. Hajnsek, "Retrieval of agricultural crop height from space: A comparison of SAR techniques," Remote Sens. Environ., vol. 187, pp. 130-144, Dec. 2016.

[10] M. I. Skolnik, "An analysis of bistatic radar," IRE Trans. Aerosp. Navigat. Electron., vol. ANE-8, no. 1, pp. 19-27, 1961.

[11] H. D. Griffiths, "From a different perspective: Principles, practice and potential of bistatic radar," in Proc. Int. Conf. Radar, Sep. 2003 pp. 1-7.

[12] C. H. Gierull, "Bistatic synthetic aperture radar, TIF-report (phase I)," Defence Res. Develop. Canada, Ottawa, ON, Canada, Tech. Rep. DRDC Ottawa TR 2004-190, 2004.

[13] A. Moccia, G. Salzillo, M. D’Errico, G. Rufino, and G. Alberti, "Performance of spaceborne bistatic synthetic aperture radar," IEEE Trans. Aerosp. Electron. Syst., vol. 41, no. 4, pp. 1383-1395, Oct. 2005

[14] A. Moccia, G. Rufino, M. D'Errico, G. Alberti, and G. Salzillo, "BISSAT: A bistatic SAR for Earth observation," in Proc. IEEE Int. Geosci. Remote Sens. Symp., vol. 5, 2002, pp. 2628-2630. [Online]. Available: http://ieeexplore.ieee.org/document/1026723/

[15] D. Massonnet, "The interferometric cartwheel: A constellation of passive satellites to produce radar images to be coherently combined," Int J. Remote Sens., vol. 22, no. 12, pp. 2413-2430, 2001. [Online]. Available: https://www.tandfonline.com/doi/full/10.1080/01431160118952

[16] H. A. Zebker, T. G. Farr, R. P. Salazar, and T. H. Dixon, "Mapping the world's topography using radar interferometry: The TOPSAT mission," Proc. IEEE, vol. 82, no. 12, pp. 1774-1786, Dec. 1994. [Online]. Available: http://ieeexplore.iee.org/document/338070/

[17] N. Gebert, B. Carnicero Dominguez, M. W. J. Davidson, M. Diaz Martin, and P. Silvestrin, "SAOCOM-CS-A passive companion to SAOCOM for single-pass L-band SAR interferometry," in Proc. 10th Eur. Conf. Synth. Aperture Radar, Jun. 2014, pp. 1-4.

[18] P. Lopez-Dekker, H. Rott, P. Prats-Iraola, B. Chapron, K. Scipal, and E. D. Witte, "Harmony: An Earth explorer 10 mission candidate to observe land, ice, and ocean surface dynamics," in Proc. IEEE Int. Geosci. Remote Sens. Symp. (IGARSS), Jul. 2019, pp. 8381-8384. [Online]. Available: https://ieeexplore.ieee.org/document/8897983/

[19] M. Davidson et al., "Copernicus L-band SAR mission requirements document," Eur. Space Agency, Paris, France, Tech. Rep. ESA-EOPSMCLIS-MRD-3371, 2019, no. 2.

[20] T. G. Farr et al., "The shuttle radar topography mission," Rev. Geophys., vol. 45, no. 2, May 2007, Art. no. RG2004, doi: 10.1029/2005rg000183.

[21] G. Krieger et al., "TanDEM-X: A satellite formation for high-resolution SAR interferometry," IEEE Trans. Geosci. Remote Sens., vol. 45, no. 11, pp. 3317-3341, Nov. 2007.

[22] A. Meta, C. Trampuz, A. Coccia, M. Ortolani, and R. Turtolo, "First results of the BelSAR L band airborne bistatic fully polarimetric synthetic aperture radar campaign," in Proc. IEEE Int. Geosci. Remote Sens. Symp. (IGARSS), Jul. 2017, pp. 1040-1042.

[23] K. A. C. de Macedo, S. Placidi, and A. Meta, "Bistatic and monostatic InSAR results with the metasensing airborne SAR system," in Proc. 6th Asia-Pacific Conf. Synth. Aperture Radar (APSAR), Nov. 2019, pp. 1-5.

[24] M. Rodriguez-Cassola, S. V. Baumgartner, G. Krieger, and A. Moreira, "Bistatic TerraSAR-X/F-SAR spaceborne-airborne SAR experiment: Description, data processing, and results," IEEE Trans. Geosci. Remote Sens., vol. 48, no. 2, pp. 781-794, Oct. 2010.

[25] I. Walterscheid, T. Espeter, A. Brenner, J. Klare, J. Ender, H. Nies, R. Wang, and O. Loffeld, "Bistatic SAR experiments with PAMIR and TerraSAR-X-setup, processing, and image results," IEEE Trans. Geosci. Remote Sens., vol. 48, no. 8, pp. 3268-3279, Aug. 2010. 
[26] K. A. Lukin et al., "Ka-band bistatic ground-based noise waveform SAR for short-range applications," IET Radar, Sonar Navigat., vol. 2, no. 4 , pp. 233-243, Aug. 2008

[27] M. Pieraccini and L. Miccinesi, "Bistatic ArcSAR," in Proc. 2nd URSI Atlantic Radio Sci. Meeting (AT-RASC), May 2018, pp. 1-4.

[28] S. Wang, W. Feng, K. Kikuta, G. Chernyak, and M. Sato, "Ground-based bistatic polarimetric interferometric synthetic aperture radar system," in Proc. IEEE Int. Geosci. Remote Sens. Symp. (IGARSS), Jul. 2019, pp. $8558-8561$.

[29] A. Anghel, R. Cacoveanu, A.-S. Moldovan, B. Rommen, and M. Datcu, "COBIS: Opportunistic C-band bistatic SAR differential interferometry" IEEE J. Sel. Topics Appl. Earth Observ. Remote Sens., vol. 12, no. 10, pp. 3980-3998, Oct. 2019. [Online]. Available: https://ieeexplore.ieee.org/document/8839119/

[30] L. Maslikowski, P. Samczynski, M. Baczyk, P. Krysik, and K. Kulpa, "Passive bistatic SAR imaging-Challenges and limitations," IEEE Aerosp. Electron. Syst. Mag., vol. 29, no. 7, pp. 23-29, Jul. 2014.

[31] S. Reuter, F. Behner, H. Nies, O. Loffeld, D. Matthes, and J. Schiller, "Development and experiments of a passive SAR receiver system in a bistatic spaceborne/stationary configuration," in Proc. IEEE Int. Geosci. Remote Sens. Symp., Jul. 2010, pp. 118-121.

[32] J. Sanz-Marcos, P. Lopez-Dekker, J. J. Mallorqui, A. Aguasca, and P. Prats, "SABRINA: A SAR bistatic receiver for interferometric applications," IEEE Geosci. Remote Sens. Lett., vol. 4, no. 2, pp. 307-311, Apr. 2007. [Online]. Available: http://ieeexplore.ieee.org/document/4156170/

[33] P. Lopez-Dekker, J. J. Mallorgui, P. Serra-Morales, and J. Sanz-Marcos, "Phase synchronization and Doppler centroid estimation in fixed receiver bistatic SAR systems," IEEE Trans. Geosci. Remote Sens., vol. 46, no. 11, pp. 3459-3471, Nov. 2008. [Online]. Available: http://ieeexplore.ieee.org/document/4685944/

[34] S. Duque, P. Lopez-Dekker, and J. J. Mallorqui, "Single-pass bistatic SAR interferometry using fixed-receiver configurations: Theory and experimental validation," IEEE Trans. Geosci. Remote Sens., vol. 48, no. 6, pp. 2740-2749, Jun. 2010. [Online]. Available: http://ieeexplore.ieee.org/document/5438816/

[35] N. J. Willis, Bistatic Radar, vol. 2. Raleigh, NC, USA: SciTech, 2005.

[36] M. Cherniakov, Bistatic Radar, M. Cherniakov, Ed. Chichester, U.K.: Wiley, Feb. 2008. [Online]. Available: http://doi.wiley.com/10. 1002/9780470985755

[37] K. Sarabandi and F. T. Ulaby, "A convenient technique for polarimetric calibration of single-antenna radar systems," IEEE Trans. Geosci. Remote Sens., vol. 28, no. 6, pp. 1022-1033, Nov. 1990.

[38] K. Sarabandi, F. T. Ulaby, and M. A. Tassouji, "Calibration of polarimetric radar systems with good polarization isolation," IEEE Trans. Geosci. Remote Sens., vol. 28, no. 1, pp. 70-75, Jan. 1990.

[39] M. Fujita, T. Masuda, Y. Fujino, and M. Satake, "Polarimetric calibration of the SIR-C C-band channel using active radar calibrators and polarization selective dihedrals," IEEE Trans. Geosci. Remote Sens., vol. 36 , no. 6, pp. $1872-1878$, Nov. 1998

[40] S. Baffelli, O. Frey, C. Werner, and I. Hajnsek, "Polarimetric calibration of the Ku-band advanced polarimetric radar interferometer," IEEE Trans. Geosci. Remote Sens., vol. 56, no. 4, pp. 2295-2311, Apr. 2018.

[41] C. Beaudoin, T. Horgan, G. Demartinis, M. J. Coulombe, A. J. Gatesman, and W. E. Nixon, "Fully polarimetric bistatic radar calibration with modified dihedral objects," IEEE Trans. Antennas Propag., vol. 66, no. 2, pp. 937-950, Feb. 2018.

[42] D. Kahny, K. Schmitt, and W. Wiesbeck, "Calibration of bistatic polarimetric radar systems," IEEE Trans. Geosci. Remote Sens., vol. 30, no. 5, pp. 847-852, Sep. 1992.

[43] C. Monzon, "A cross-polarized bistatic calibration device for RCS measurements," IEEE Trans. Antennas Propag., vol. 51, no. 4, pp. 833-839, Apr. 2003.

[44] D. R. Brunfeldt and F. T. Ulaby, "Active reflector for radar calibration," IEEE Trans. Geosci. Remote Sens., vol. GE-22, no. 2, pp. 165-169, Mar. 1984

[45] M. Pienaar, J. W. Odendaal, J. Joubert, J. E. Cilliers, and J. C. Smit, "Active calibration target for bistatic radar cross-section measurements," Radio Sci., vol. 51, no. 5, pp. 515-523, May 2016.
[46] S. Baffelli, O. Frey, and I. Hajnsek, "Polarimetric analysis of natural terrain observed with a $K u$-band terrestrial radar," IEEE J. Sel. Topics Appl. Earth Observ. Remote Sens., vol. 12, no. 12, pp. 5268-5288, Dec. 2019. [Online]. Available: https://ieeexplore.ieee.org/document/ $8960552 /$

[47] S. Baffelli, O. Frey, and I. Hajnsek, "Geostatistical analysis and mitigation of the atmospheric phase screens in Ku-band terrestrial radar interferometric observations of an Alpine glacier," IEEE Trans. Geosci. Remote Sens., vol. 58, no. 11, pp. 7533-7556, Nov. 2020. [Online]. Available: https://ieeexplore.ieee.org/document/9064718/

[48] C. Werner, A. Wiesmann, T. Strozzi, A. Kos, R. Caduff, and U. Wegmuller, "The GPRI multi-mode differential interferometric radar for ground-based observations," in Proc. 9th Eur. Conf. Synth. Aperture Radar, Apr. 2012, pp. 304-307.

[49] T. Strozzi, C. Werner, A. Wiesmann, and U. Wegmuller, "Topography mapping with a portable real-aperture radar interferometer," IEEE Geosci. Remote Sens. Lett., vol. 9, no. 2, pp. 277-281, Mar. 2012.

[50] R. Caduff, A. Kos, F. Schlunegger, B. W. McArdell, and A. Wiesmann, "Terrestrial radar interferometric measurement of hillslope deformation and atmospheric disturbances in the illgraben debris-flow catchment, Switzerland," IEEE Geosci. Remote Sens. Lett., vol. 11, no. 2, pp. 434-438, Feb. 2014. [Online]. Available: http://ieeexplore.ieee.org/document/6558751/

[51] A. Wiesmann, R. Caduff, and C. Mätzler, "Terrestrial radar observations of dynamic changes in Alpine snow," IEEE J. Sel. Topics Appl. Earth Observ. Remote Sens., vol. 8, no. 7, pp. 3665-3671, Jul. 2015. [Online]. Available: http://ieeexplore.ieee.org/document/7059220/

[52] M. Rodriguez-Cassola et al., "First bistatic spaceborne SAR experiments with TanDEM-X," IEEE Geosci. Remote Sens. Lett., vol. 9, no. 1, pp. 33-37, Jan. 2012.

[53] A. G. Stove, "Linear FMCW radar techniques," IEE Proc. F (Radar Signal Process.), vol. 139, no. 5, pp. 343-350, 1992.

[54] E. Rodriguez and J. M. Martin, "Theory and design of interferometric synthetic aperture radars," IEE Proc. F (Radar Signal Process.), vol. 139, no. 2, pp. 147-159, 1992.

[55] D. Just and R. Bamler, "Phase statistics of interferograms with applications to synthetic aperture radar," Appl. Opt., vol. 33, no. 20, p. 4361, Jul. 1994. [Online]. Available: https://www.osapublishing. org/abstract.cfm?URI=ao-33-20-4361

[56] S. M. Kay, Fundamentals of Statistical Signal Processing: Estimation Theory. Upper Saddle River, NJ, USA: Prentice-Hall, 1993.

[57] N. A. Department, Electronic Warfare and Radar Systems Engineering Handbook. New York, NY, USA: MTTS \& IEEE, p. 455, 1997.

[58] M. C. Budge and S. R. German, Basic Radar Analysis. Norwood, MA, USA: Artech House, 2015.

[59] M. M. Weiner and P. Kaplan, "Bistatic surface clutter resolution area at small grazing angles," MITRE CORP, Bedford, MA, USA, Tech. Rep. RADC-TR-82-289, 1982.

[60] C. J. Bradley et al., "An investigation of bistatic calibration techniques," IEEE Trans. Geosci. Remote Sens., vol. 43, no. 10, pp. 2185-2191, Oct. 2005.

[61] G. Krieger, I. Hajnsek, K. Papathanassiou, M. Younis, and A. Moreira, "Interferometric synthetic aperture radar (SAR) missions employing formation flying," Proc. IEEE, vol. 98, no. 5, pp. 816-843, May 2010.

[62] Y. Inoue et al., "Season-long daily measurements of multifrequency $(\mathrm{Ka}, \mathrm{Ku}, \mathrm{X}, \mathrm{C}$, and $\mathrm{L}$ ) and full-polarization backscatter signatures over paddy rice field and their relationship with biological variables," Remote Sens. Environ., vol. 81, nos. 2-3, pp. 194-204. 2002.

[63] E. Erten, C. Rossi, and O. Yuzugullu, "Polarization impact in TanDEM-X data over vertical-oriented vegetation: The paddy-rice case study," IEEE Geosci. Remote Sens. Lett., vol. 12, no. 7, pp. 1501-1505, Jul. 2015.

[64] E. Akkermans, P. Wolf, and R. Maynard, "Coherent backscattering of light by disordered media: Analysis of the peak line shape," Phys. Rev. Lett., vol. 56, no. 14, p. 1471, 1986.

[65] S. Tan, W. Chang, L. Tsang, J. Lemmetyinen, and M. Proksch, "Modeling both active and passive microwave remote sensing of snow using dense media radiative transfer (DMRT) theory with multiple scattering and backscattering enhancement," IEEE J. Sel. Topics Appl. Earth Observ. Remote Sens., vol. 8, no. 9, pp. 4418-4430, Sep. 2015. 


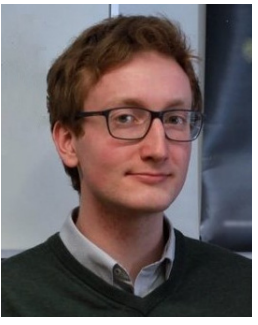

Marcel Stefko (Student Member, IEEE) received the Bc. degree in physical engineering and nanotechnology from the Brno University of Technology, Brno, Czech Republic, in 2015, and the M.Sc. degree in applied physics from the École Polytechnique Fédérale de Lausanne (EPFL), Lausanne, Switzerland, in 2017. He is currently pursuing the Ph.D. degree with the Chair of Earth Observation and Remote Sensing, ETH Zürich, Zürich, Switzerland

From 2017 to 2018, he worked as a Young Graduate Trainee with the European Space Agency, Noordwijk, The Netherlands. His research interests include development of calibration, data acquisition, and data processing methods for bistatic radar systems, and applications of these systems toward monitoring of natural environments.

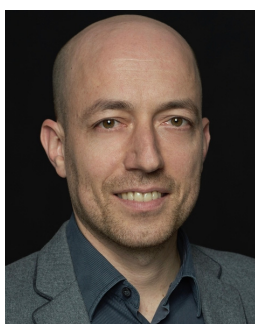

Othmar Frey (Senior Member, IEEE) received the M.Sc. degree (ETH Medal awarded for M.Sc Thesis) in geomatic engineering from ETH Zürich, Zürich, Switzerland, in 2002, and the Ph.D. (Dr.sc.nat.) degree (Distinction Award and Prize from the Faculty of Science) in radar remote sensing from the University of Zurich, Zürich, in 2010.

$\mathrm{He}$ has been working in this joint academic/ industry setting since 2011. From 2002 to 2010, he was a Research Associate with the Remote Sensing Laboratory, University of Zurich. He is currently a tenured Senior Scientist and a Lecturer with ETH Zürich and a Senior Scientist with GAMMA Remote Sensing AG, Gümligen, Switzerland. His research encompasses 2-D and 3-D (tomographic) synthetic aperture radar (SAR) imaging and interferometric/polarimetric techniques for applications, such as 3-D forest mapping, ground deformation monitoring, and snow layer profiling using spaceborne, airborne, UAV-borne, and terrestrial radar sensors. $\mathrm{He}$ has been a PI/co-investigator in various national and international research projects. Recently, he has been a PI of an Innosuisse project in which a new car-borne InSAR system for mobile mapping of surface displacements has been developed. At GAMMA, he has also been active in the development of the Gamma Software and in technical/scientific consulting on SAR imaging and interferometric applications to ESA, NASA, and aerospace industry.

Dr. Frey was a member of the SAOCOM-CS Science Expert Group with the European Space Agency. Since 2015, he has been the Chair of the Swiss Chapter of the IEEE Geoscience and Remote Sensing Society.

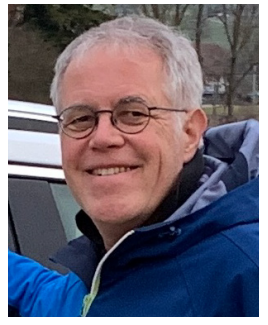

Charles Werner (Life Senior Member, IEEE) received the M.S. degree in electrical engineering and the Ph.D. degree in systems engineering from the University of Pennsylvania, Philadelphia, PA, USA, in 1982, and 1987, respectively. His Ph.D. work involved tomographic 3-D microwave and acoustic imaging.

From 1982 until 1999, he was employed at the Jet Propulsion Laboratory, Pasadena, CA, USA, for radar system engineering of Earth and planetary SAR systems, including the Cassini radar instrument and the shuttle topographic mapping radar (SRTM) missions. While a Visiting Researcher at the University of Zurich, Zürich, Switzerland, from 1993 to 1995, he developed an end-to-end interferometric radar processing system for airborne and spaceborne SAR. Together with Urs Wegmüller, in 1995, they founded Gamma Remote Sensing AG, Gümligen, Switzerland. He is currently involved in the development of Gamma's radar application software for calculation of interferometric deformation time-series, terrain geocoding, and visualization. He is also active in the development, design, and production of microwave instrumentation, including ground-based Ku-band real-aperture radars for near real-time deformation monitoring, wideband microwave scatterometers, and multichannel interferometric synthetic aperture radars.

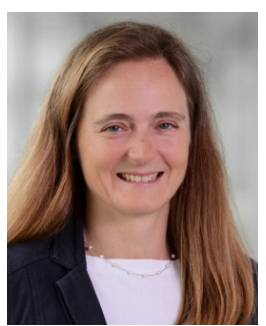

Irena Hajnsek (Fellow, IEEE) received the Dipl. degree (Hons.) in fluvial river systems from the Free University of Berlin, Berlin, Germany, in 1996, and the Dr. rer. nat. degree (Hons.) in model-based estimation of soil moisture from fully polarimetric synthetic aperture radar from the Friedrich Schiller University of Jena, Jena, Germany, in 2001.

Since November 2009, she has been a Professor of Earth observation with the Swiss Federal Institute of Technology (ETH) Zürich, Institute of Environmental Engineering, and at the same time Head of the Polarimetric SAR Interferometry research group, Microwaves and Radar Institute, German Aerospace Center, Weßling, Germany. Since 2010, she has been the Science Coordinator of the German Satellite Mission TanDEM-X and proposed satellite mission Tandem-L. Her research interests include electromagnetic propagation and scattering theory, radar polarimetry, SAR and interferometric SAR data processing techniques, and environmental parameter modeling and estimation.

Dr. Hajnsek is currently a member of the European Space Agency Mission Advisory Group for the ROSE-L Mission. She was the Technical Program Co-Chair of the IEEE International Geoscience and Remote Sensing Symposium (IGARSS) 2012, Munich, Germany, and the IEEE IGARSS 2019, Yokohama, Japan. Since 2013, she has been a member of the IEEE Geoscience and Remote Sensing Society (GRSS) AdCom. From 2016 to 2020, she was the Vice President of the IEEE GRSS Technical Committee. 